\title{
Measuring gravitational-wave higher-order multipoles
}

\author{
Cameron Mills $\odot$ and Stephen Fairhurst $\odot$ \\ Gravity Exploration Institute, School of Physics and Astronomy, Cardiff University, \\ The Parade, Cardiff CF24 3AA, United Kingdom
}

(Received 6 August 2020; accepted 8 December 2020; published 21 January 2021)

\begin{abstract}
We investigate the observability of higher harmonics in gravitational wave signals emitted during the coalescence of binary black holes. We decompose each harmonic into an overall amplitude, dependent upon the masses and spins of the system, and an orientation-dependent term, dependent upon the inclination and polarization of the source. Using this decomposition, we investigate the significance of higher multipole moments over the parameter space and show that the $\ell=|m|=3$ harmonic is most significant across much of the sensitive band of ground-based interferometric detectors, with the $\ell=|m|=4$ harmonic having a significant contribution at high masses. We introduce the higher harmonic signal-to-noise ratio (SNR), and show that a simple threshold on this SNR can be used as a criterion for observation of higher harmonics. Finally, we investigate observability in a population of binaries and show that higher harmonics will be observable in a few percent of binaries, typically those with unequal masses and viewed close to edge-on. We find that he majority of binaries with mass ratio greater than $4: 1$ will have an observable $\ell=|m|=3$ harmonic.
\end{abstract}

DOI: 10.1103/PhysRevD.103.024042

\section{INTRODUCTION}

Gravitational waves emitted during the coalescence of black hole and/or neutron star binaries are emitted predominantly at twice the orbital frequency, during the inspiral phase of the coalescence [1]. However, it is also well known that the gravitational wave signal cannot be completely characterized by a single harmonic but, rather, is better decomposed as a sum of spin-weighed spherical [2,3] (or spheroidal [4-8]) harmonics. The dominant harmonic is the $(\ell,|m|)=(2,2)$ harmonic, but there is also power in higher harmonics, most notably the $(\ell,|m|)=(2,1),(3,2),(3,3)$ and $(4,4)$ harmonics ${ }^{1}[9,10]$. The importance of these additional harmonics increases as the mass ratio between the two black holes increases and also increases for observed signals where the late inspiral and merger of the objects contribute significantly to the SNR [10-28]. Recent semianalytical and numerical relativity models have provided expressions for an increasing number of the higher harmonics accurate across the inspiral, merger and ringdown regimes [8,29-41].

Clear evidence of higher gravitational-wave harmonics has been observed in two recent observations, GW190412 [42] and GW190814 [43], as well as weaker evidence in the high-mass system GW170729 [44]. These observations provide further evidence that Einstein's general relativity is an accurate description of gravity, including in the strongfield, highly dynamic regime of the merger of two black

\footnotetext{
${ }^{1}$ When we refer to a multipole by the label $(\ell, m)$ we always mean $(\ell,|m|)$.
}

holes $[9,45]$. By incorporating knowledge of the higher harmonics into a search for gravitational waves, the sensitivity of gravitational wave searches can be improved, leading to an increase in the rate of observed systems [23]; furthermore these observations would typically be from less densely populated regions of the parameter space [46], for example high mass binaries and those with unequal mass components. Finally, the observation of higher harmonics enables more accurate measurement of the properties of system $[42,43,47]$. For example, the measurement of multiple harmonics can be used to break wellknown degeneracies between the measured distance and orientation of the system [48], or the mass ratio and spins of the black holes $[49,50]$.

The observation of other features in the gravitational waveform, most notably spin-induced orbital precession in black-hole binaries [51,52] and matter effects in binaries containing neutron stars [53,54], will also enable more accurate measurements of the source properties. Measurements of neutron-star structure have been inferred from GW170817 [55,56], while there is some evidence for precession in the black hole binary mergers GW190521 [57] and GW190412 [42]. The inclusion of matter effects will not affect the applicability of the spin-weighted spherical harmonic decomposition discussed above, and we do not consider these effects in this paper.

Orbital precession does change the structure of the emitted gravitational waveform. The waveform can still be decomposed into a basis of spin-weighted spherical harmonics, but now each of the harmonics shows the 
characteristic amplitude and phase modulations associated with time-dependent evolution of the orbital plane [32]. Alternatively, this can be understood as the splitting of each gravitational wave multipole into several precessioninduced harmonics, which are offset by multiples of the precession frequency $[32,58,59]$. Thus, a full analysis of the observability of higher multipole moments requires a detailed treatment of precession. However, for the majority of events observed to date [60], there is little, if any, evidence for precession. In this paper, we restrict attention to aligned-spin systems, which do not exhibit precession. This will provide good insight into the significance of higher gravitational wave multipoles in the observed population.

While the gravitational waveform is comprised of an infinite number of harmonics, it is the unambiguous measurement of a second harmonic, in addition to the $(\ell,|m|)=(2,2)$ harmonic, which will lead to a stepchange in our ability to measure the properties of the system; additional harmonics will then further refine the measurement accuracy. In this paper, we perform an indepth investigation of the importance of the higher harmonics across the parameter space and identify regions of the parameter space where particular harmonics are most likely to make a significant contribution. The amplitude of each harmonic depends both upon the intrinsic parameters of the system (its masses and spins, both magnitudes and orientations) as well as the extrinsic parameters (the orientation of the binary and the detector network's sensitivity to the two polarizations of gravitational waves). For simplicity, we decompose the harmonics into an overall amplitude factor, dependent only upon the intrinsic parameters, and an orientation dependent term. We then investigate the significance of each harmonic across the parameter space.

Next, we turn to the question of when additional harmonics have been unambiguously observed. From a model selection perspective, this can be addressed by considering the evidence in favour of a waveform containing higher harmonics against one without. Here, we introduce the higher-harmonic signal to noise ratio, and argue that it can be used as an alternative method of establishing the observability of higher harmonics. ${ }^{2}$ It is straightforward to calculate the SNR contained in each of the higher waveform harmonics, and compare to the expectation due to noise-only in the higher harmonics. This approach has been used to verify the observation of higher harmonics from the binary mergers observed as GW190412 and GW190814 [42,43].

The structure of the paper is as follows. In Sec. II, we provide a brief review of the gravitational waveform, incorporating the higher harmonics, and use this to fix

\footnotetext{
${ }^{2} \mathrm{~A}$ similar prescription has recently been introduced for precessing systems $[59,61]$.
}

the notation for the remainder of the paper; in Sec. III we explore the significance of the higher harmonics over the parameter space, both intrinsic (masses and spins) and extrinsic (binary orientation); in Sec. IV we investigate the observability of higher harmonics and introduce a simple criterion for detection; finally in Sec. V we investigate observability for a population of events.

\section{THE GRAVITATIONAL WAVEFORM}

The measured gravitational wave strain $h$ can be written as

$$
h=F_{+} h_{+}+F_{\times} h_{\times},
$$

where the antenna factors $F_{+}$and $F_{\times}$depend upon the sky location (right-ascension and declination) of the source, as well as the polarization of the source. It is often convenient to explicitly extract the unknown polarization angle $\psi$ and then consider the detector response to be a known quantity dependent upon only the details of the detector and the direction to the source. Thus, we write the detector response as,

$$
\begin{aligned}
& F_{+}=w_{+} \cos 2 \psi+w_{\times} \sin 2 \psi, \\
& F_{\times}=-w_{+} \sin 2 \psi+w_{\times} \cos 2 \psi,
\end{aligned}
$$

where $w_{+}$and $w_{\times}$are the detector response functions in a fixed frame-for a single detector it is natural to choose $w_{\times}=0$ and for a network to work in the dominant polarization, in which for each sky point the polarization angle $\chi$ is chosen to maximize the network sensitivity to $w_{+}$ $[62,63]$. The relative amplitude of $w_{\times}$to $w_{+}$describes the sensitivity of the network to the second gravitational wave polarization. The unknown polarization of the source relative to this preferred frame is denoted $\psi$.

The radiation-frame gravitational wave polarizations $h_{+}$ and $h_{\times}$can be decomposed into multipole moments using spin-weighted spherical harmonics of spin weight -2 , ${ }_{-2} Y_{\ell m}$, which are functions of the inclination angle $\imath$ and a reference phase $\phi_{o}$ (see the Appendix A for a more detailed discussion of the decomposition).

For binaries with aligned spins, the orbital plane remains unchanged during the merger, and this provides a natural fixed basis for the spherical harmonic decomposition. However, if spin-induced precession is present, the orbital plane changes during the course of the merger (equivalently, the inclination angle becomes time dependent). In this case, it is natural to consider the waveform in a coprecessing frame, i.e., a frame which is locked to the binary's orbit $[58,64]$. While it is still possible to decompose as a series of spin-weighted spherical harmonics, each multipole moment of the waveform in the observer's frame involves a sum over multiple harmonics in the coprecessing frame [32]. In this paper, we restrict attention to binaries which do not exhibit 
precession. While we do expect generic black hole binaries to have spins which are misaligned with the orbital angular momentum (and hence will precess), in the majority of observations to date [60] there has been little evidence for precession and low black hole spins are inferred from current observations. Furthermore, as discussed in [59], the $(2,2)$ multipole moment of a precessing waveform can be decomposed into five precession harmonics with the two leading harmonics providing the majority of the signal power. A similar decomposition is possible for the higher $(\ell,|m|)$ multipoles and, over much of the parameter space, it is only the leading precession harmonic of the higher $(\ell,|m|)$ multipoles that will provide significant power. Consequently, for many signals with nonzero in-plane spins, our analysis will remain valid. We leave the detailed examination of the interplay between precession and higher harmonics to future investigations.

For a binary merger which does not exhibit precession, the waveform can be expressed in the frequency domain, using the stationary-phase approximation, as

$$
\begin{aligned}
& \tilde{h}_{+}(f)=\frac{d_{o}}{d_{L}} \sum_{\ell \geq 2} \sum_{m=0}^{\ell} A_{+}^{\ell m}(\imath) e^{i m \phi_{o}} \tilde{h}_{\ell m}(f) \\
& \tilde{h}_{\times}(f)=\frac{d_{o}}{d_{L}} \sum_{\ell \geq 2} \sum_{m=0}^{\ell} A_{\times}^{\ell m}(\imath) i e^{i m \phi_{o}} \tilde{h}_{\ell m}(f)
\end{aligned}
$$

where $d_{L}$ is the luminosity distance, $d_{o}$ is a fiducial distance used to normalize the waveforms $\tilde{h}_{\ell m}$. The amplitude factors $A^{\ell m}$ are functions only of the inclination angle and are given below for the most significant harmonics:

$$
\begin{aligned}
& A_{+}^{22}=\frac{1}{2}\left(1+\cos ^{2} \imath\right) \\
& A_{\times}^{22}=\cos \imath \\
& A_{+}^{21}=\sin \imath \\
& A_{\times}^{21}=\sin \imath \cos \imath \\
& A_{+}^{33}=\sin \imath\left(1+\cos ^{2} \imath\right) \\
& A_{\times}^{33}=2 \sin \imath \cos \imath \\
& A_{+}^{32}=1-2 \cos ^{2} \imath \\
& A_{\times}^{32}=\frac{1}{2}\left(\cos \imath-3 \cos ^{3} \imath\right) \\
& A_{+}^{44}=\sin ^{2} \imath\left(1+\cos ^{2} \imath\right) \\
& A_{\times}^{44}=2 \sin ^{2} \imath \cos \imath
\end{aligned}
$$

There is a freedom in choice of overall normalization for these amplitude factors, which corresponds to an overall rescaling of the waveform defining each harmonic, $\tilde{h}_{\ell m}$. For the $(\ell,|m|)=(2,2)$ multipole moment, it is customary to choose a normalization such that $A_{+}^{22}=A_{\times}^{22}=1$ for a face-on system, and we use that normalization here. Since many of the higher harmonics vanish for face-on systems, we instead choose a normalization for the higher-harmonic amplitude factors, $A_{+, \times}^{\ell m}$ in Eq. (4), by requiring that for the plus polarization $A_{+}^{\ell m}=1$ at $l=\frac{\pi}{2}$, i.e., when the system is edge on. ${ }^{3}$

Fig. 1 shows the dependence of the multipole moments on inclination. The plus polarization of the $(2,2)$ harmonic peaks at face-on, while the $(2,1)$ and $(4,4)$ harmonics peak at edge-on. The $(3,2)$ amplitude factor is maximum at both face-on and edge-on orientations while the $(3,3)$ harmonic peaks at $\sin l=\sqrt{\frac{2}{3}}$. The different dependence of the harmonics on the binary orientation can lead to the improved measurement of the inclination, when more than one harmonic is observed [42,47], breaking the well-known degeneracy between distance and inclination angle that arises when observing only the dominant harmonic [48].

During inspiral, the frequency evolution of a multipole, $\omega_{\ell m}$, is related to the orbital frequency, $\omega_{\text {orb }}$, as $\omega_{\ell m} \sim$ $m \omega_{\text {orb }}$ [9]. Phenomenologically, it has been qualitatively observed that during the ringdown the frequency approximately evolves as $\omega_{\ell m} \sim l \omega_{\text {orb }}[5,65]$. Thus it is possible to scale the frequencies of the $(2,2)$ multipole moment in quite a simple manner to obtain an approximate phase evolution of the $l=m$ harmonics, for example the phase evolution of the $(3,3)$ multipole moment is approximately a factor of 1.5 times $\omega_{22}$ [66].

\section{THE SIGNIFICANCE OF HIGHER HARMONICS}

In this section, we investigate the observability of the different $(\ell, m)$ multipole moments, and how this varies across the mass and spin parameter space. For concreteness, we restrict attention to a single detector with a sensitivity comparable to that achieved by the LIGO observatories during their third observing run [67].

The key metric for waveform observability is the optimal signal-to-noise ratio (SNR) defined as

$$
\hat{\rho}=\sqrt{(h \mid h)}
$$

where we have introduced the inner product weighted by noise (characterized by a power spectrum $S(f)$ ) as

$$
(a \mid b):=4 \operatorname{Re} \int_{0}^{f_{\max }} \frac{\tilde{a}(f) \tilde{b}(f)^{\star}}{S(f)} \mathrm{d} f .
$$

\footnotetext{
${ }^{3}$ The normalization obtained by directly expanding the spherical harmonics from Eq. (A2) in Eq. (A6) differs by multiplicative factors of $\frac{1}{4} \sqrt{\frac{5}{\pi}}, \frac{1}{4} \sqrt{\frac{5}{\pi}},-\frac{1}{8} \sqrt{\frac{21}{2 \pi}},-\frac{1}{4} \sqrt{\frac{7}{\pi}}$ and $\frac{3}{16} \sqrt{\frac{7}{\pi}}$ for the $(2,2)$, $(2,1),(3,3),(3,2)$ and $(4,4)$ multipoles respectively. The calculation is presented in Appendix A.
} 

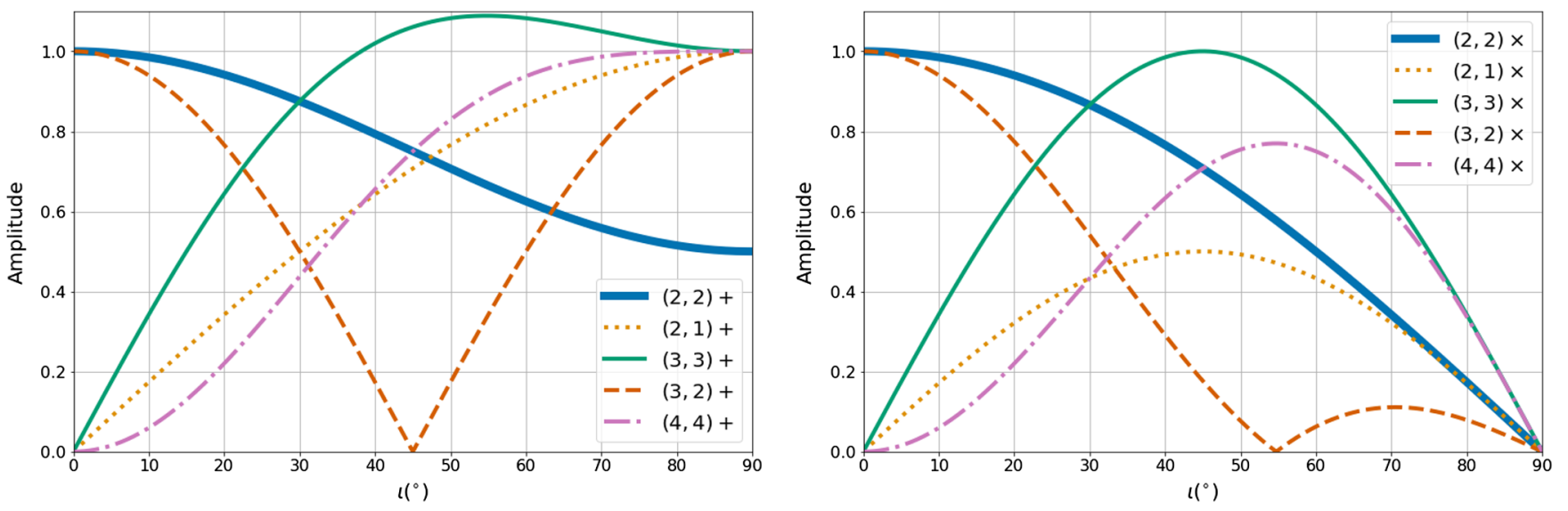

FIG. 1. The absolute amplitude factors of the $(2,2),(2,1),(3,3),(3,2)$ and $(4,4)$ harmonics as a function of the inclination $\imath$ of the binary. The $(2,2)$ multipole moment is normalized to unity at $l=0^{\circ}$ while other multipoles are normalized to unit amplitude in the + polarization at $\iota=90^{\circ}$. Left: +polarization, Right: $\times$ polarization.

Consider the situation where the $(\ell,|m|)=(2,2)$ harmonic has been observed, and we are interested in obtaining an estimate of the expected SNR in the other harmonics. As is clear from Eq. (3), the SNR in the higher harmonics will depend upon the detector sensitivity to the higher harmonic waveform, $\tilde{h}_{\ell m}$, as well as the amplitude factor $A_{+, \times}^{\ell m}$.

Let us examine the single-detector case in detail. For simplicity, we choose a detector sensitive only to the + polarization (in the preferred frame), so that $w_{\times}=0$, and we take $w_{+}=1$. Furthermore, we simplify the calculation to consider only two multipole moments, the $(2,2)$ harmonic and one other generic $(\ell, m)$ harmonic. The amplitude of each multipole depends on both the intrinsic properties of the system and the orientation relative to the network of detectors.

The waveform observed at the detector is

$$
h=\cos 2 \psi\left(h_{+}^{22}+h_{+}^{\ell m}\right)-\sin 2 \psi\left(h_{\times}^{22}+h_{\times}^{\ell m}\right),
$$

where $h_{+, \times}^{\ell m}$ are the two orthogonal components for the $(\ell, m)$ multipole moment of the waveform. For the explicit frequency domain decomposition introduced in Eq. (3), we can write

$$
\begin{aligned}
& \tilde{h}_{+}^{\ell m}(f)=\frac{d_{o}}{d_{L}} A_{+}^{\ell m}(\imath) e^{i m \phi_{o}} \tilde{h}_{\ell m}(f) \\
& \tilde{h}_{\times}^{\ell m}(f)=\frac{d_{o}}{d_{L}} A_{\times}^{\ell m}(\imath) i e^{i m \phi_{o}} \tilde{h}_{\ell m}(f)
\end{aligned}
$$

using the fact that $F_{+}=\cos 2 \psi, F_{\times}=-\sin 2 \psi$.

A simple substitution of Eq. (7) into Eq. (6) gives the optimal SNR for a signal comprising two harmonics as

$$
\begin{aligned}
\hat{\rho}^{2}= & \cos ^{2} 2 \psi\left[\left|\tilde{h}_{+}^{22}\right|^{2}+\left|\tilde{h}_{+}^{\ell m}\right|^{2}+2\left(\tilde{h}_{+}^{\ell m} \mid \tilde{h}_{+}^{22}\right)\right] \\
& +\sin ^{2} 2 \psi\left[\left|\tilde{h}_{\times}^{22}\right|^{2}+\left|\tilde{h}_{\times}^{\ell m}\right|^{2}+2\left(\tilde{h}_{\times}^{\ell m} \mid \tilde{h}_{\times}^{22}\right)\right] \\
& -2 \sin 2 \psi \cos 2 \psi\left[\left(\tilde{h}_{+}^{22} \mid \tilde{h}_{\times}^{\ell m}\right)+\left(h_{\times}^{22} \mid h_{+}^{\ell m}\right)\right]
\end{aligned}
$$

where the cross terms $(\sin 2 \psi \cos 2 \psi)$ between polarizations for a single mode cancel since $\left(\tilde{h}_{+}^{\ell m} \mid \tilde{h}_{\times}^{\ell m}\right)=0$.

The cross terms between different multipole moments, $\left(\tilde{h}_{+, \times}^{\ell m} \mid \tilde{h}_{+, \times}^{22}\right)$, can be both positive or negative, causing constructive or destructive interference between the harmonics. As discussed previously, the frequency during inspiral scales with $m$ while the ringdown frequency has been observed to scale approximately with $\ell$. Consequently, there is typically little overlap between the $(2,2)$ multipole and multipoles for which both $\ell \neq 2$ and $m \neq 2$. Thus, in many cases, the cross terms between different harmonics will not make a significant contribution. We examine in detail the importance of the overlap between different multipole moments in Sec. III A. For now, we restrict to the case where these terms can be neglected.

Neglecting the cross terms between harmonics, $\left(\tilde{h}_{+, \times}^{\ell m} \mid \tilde{h}_{+, \times}^{22}\right)$, we are able to reexpress the optimal SNR as

$$
\begin{aligned}
\hat{\rho}^{2}= & \left(\cos ^{2} 2 \psi\left|\tilde{h}_{+}^{22}\right|^{2}+\sin ^{2} 2 \psi\left|\tilde{h}_{\times}^{22}\right|^{2}\right) \\
& +\left(\cos ^{2} 2 \psi\left|\tilde{h}_{+}^{\ell m}\right|^{2}+\sin ^{2} 2 \psi\left|\tilde{h}_{\times}^{\ell m}\right|^{2}\right) \\
= & \rho_{22}^{2}+\rho_{\ell m}^{2} .
\end{aligned}
$$

We have defined, in the obvious way, the power in the $(2,2)$ and $(\ell, m)$ multipole moments as $\rho_{22}^{2}$ and $\rho_{\ell m}^{2}$ respectively.

Next, using the form of $h_{+, \times}^{\ell m}$ from Eq. (8), we can write

$$
\rho_{\ell m}^{2}=\left[\left(A_{+}^{\ell m}\right)^{2} \cos ^{2} 2 \psi+\left(A_{\times}^{\ell m}\right)^{2} \sin ^{2} 2 \psi\right]\left|\tilde{h}_{\ell m}\right|^{2},
$$

which is also valid for $(\ell, m)=(2,2)$. The SNR in the $(\ell, m)$ harmonic is given by an orientation contribution, 
dependent upon inclination and polarization, and a term that depends upon the overall amplitude of the given multipole moment. Consequently, we can express the SNR in the higher harmonic as:

$$
\rho_{\ell m}=\rho_{22} \alpha_{\ell m} R_{\ell m},
$$

where $\alpha_{\ell m}$ encodes the relative, intrinsic amplitude of the $(\ell, m)$ multipole moment relative to the $(2,2)$ multipole and $R_{\ell, m}$ encodes the relative size of the orientation factors. Specifically,

$$
\alpha_{\ell m}^{2}=\frac{\left(\tilde{h}_{\ell m} \mid \tilde{h}_{\ell m}\right)}{\left(\tilde{h}_{22} \mid \tilde{h}_{22}\right)}
$$

and

$$
R_{\ell m}^{2}=\frac{\left[\left(A_{+}^{\ell m}\right)^{2} \cos ^{2} 2 \psi+\left(A_{\times}^{\ell m}\right)^{2} \sin ^{2} 2 \psi\right]}{\left[\left(A_{+}^{22}\right)^{2} \cos ^{2} 2 \psi+\left(A_{\times}^{22}\right)^{2} \sin ^{2} 2 \psi\right]} .
$$

In general, the relative amplitudes $R_{\ell m}$ will depend upon both the inclination and polarization angles. However, for the $\ell=m$ multipole moments, the expression simplifies as the relative amplitudes are the same for both,$+ \times$ polarization. In this case, there is no dependence upon the polarization angle and ${ }^{4}$

$$
\begin{aligned}
& R_{33}(l)=2 \sin \imath \\
& R_{44}(l)=2 \sin ^{2} \imath .
\end{aligned}
$$

In Sec. III A, we explore the dependence of the relative amplitudes $\alpha_{\ell m}$ over the mass and spin parameter space and, in Sec. III B, the expected distribution of $R_{\ell m}$ for a population of sources.

\section{A. Dependence upon intrinsic parameters}

The two important intrinsic parameters determining the relative power in the higher harmonics are mass ratio and total mass, with spin effects entering at higher postNewtonian (PN) order for most harmonics [10]. The contribution of a higher harmonic relative to the $(2,2)$ harmonic generically increases with an increasing mass ratio. The relative amplitudes of the multipole moments are independent of the total mass of the system. However the frequency content of each multipole does depend upon the total mass and thus, depending on the shape of the detector power spectral density, certain higher harmonics might be preferentially observed. In particular, the contribution of higher harmonics can become more significant at high masses, for which the merger frequency of the dominant harmonic lies below the optimal sensitivity of the detector.

\footnotetext{
${ }^{4}$ In this case, the cross term between polarizations in Eq. (9) also vanishes.
}

In Fig. 2 we show the relative amplitude, $\alpha_{l m}$, of the four multipoles that we are considering: the $(3,3),(4,4),(2,1)$ and $(3,2)$ harmonics. The amplitudes have been calculated using the PhenomHM waveform [30], for a signal observed in a detector with LIGO O3 sensitivity [60,67], as a function of the (detector frame) total mass and mass ratio of the system.

Over much of the parameter space, the $(3,3)$ harmonic is the most significant, with the relative amplitude of the $(3,3)$ harmonic increasing with mass ratio. For example, at a total mass of $50 M_{\odot}$, the $(3,3)$ harmonic has $10 \%$ of the amplitude of the leading harmonic at a mass ratio of $2: 1$ and $20 \%$ at $5: 1$. At high masses, and significant mass ratios, the relative sensitivity to the $(3,3)$ harmonic is greater than one third of the $(2,2)$ multipole. The $(4,4)$ multipole is generally the third most significant, after the $(2,2)$ and $(3,3)$ harmonics. However, sensitivity to the $(4,4)$ multipole increases rapidly as the mass of the system increases so that for total mass above $\sim 75 M_{\odot}$ and mass ratio less than $2: 1$, the $(4,4)$ multipole is more significant than the $(3,3)$ multipole.

The intrinsic amplitudes of the $(2,1)$ and $(3,2)$ harmonics are always lower than at least one of the $(3,3)$ and $(4,4)$ harmonics, over the mass and mass ratio ranges explored in Fig. 2. As with the other harmonics, their relative importance increases as the mass ratio decreases and also, for the $(3,2)$ harmonic in particular, as the total mass increases. The $(2,1)$ multipole is the only subdominant multipole considered in this paper which has spin terms in the amplitude at 1 PN order [10]. For this reason, the $(2,1)$ multipole is more significant for binaries with large antialigned spins: the intrinsic amplitude roughly doubles for a binary with effective spin $\chi_{\text {eff }}=-0.8$, relative to a nonspinning system. Even then, the relative contribution of the $(2,1)$ harmonic is less than the $(3,3)$ harmonic.

For the power in these higher harmonics to be observable, it must be possible to distinguish the signal in the higher harmonic from the $(2,2)$ harmonic. Generally, it is only the contribution which is orthogonal to the $(2,2)$ harmonic which will be observable. Any contribution from the higher harmonics which is proportional to the $(2,2)$ harmonic will simply serve to change the power observed in the $(2,2)$. Consequently, we are interested in knowing whether the waveforms are orthogonal or, equivalently, in the overlap between the harmonics. Here, we define the normalized overlap maximized over the reference phase, $\phi_{o}$,

$$
O(\ell m, 22)=\frac{\operatorname{Max}_{\phi_{o}}\left(\tilde{h}_{\ell m} \mid \tilde{h}_{22}\right)}{\left|\tilde{h}_{\ell m}\right|\left|\tilde{h}_{22}\right|} .
$$

The overlap between the $(3,3)$ and $(4,4)$ multipoles with the $(2,2)$ harmonic is $<10 \%$ across the parameter space explored, as expected due to the fact that the frequency evolution of these harmonics differs significantly from the $(2,2)$. However, the overlap of the $(2,2)$ harmonic with the 

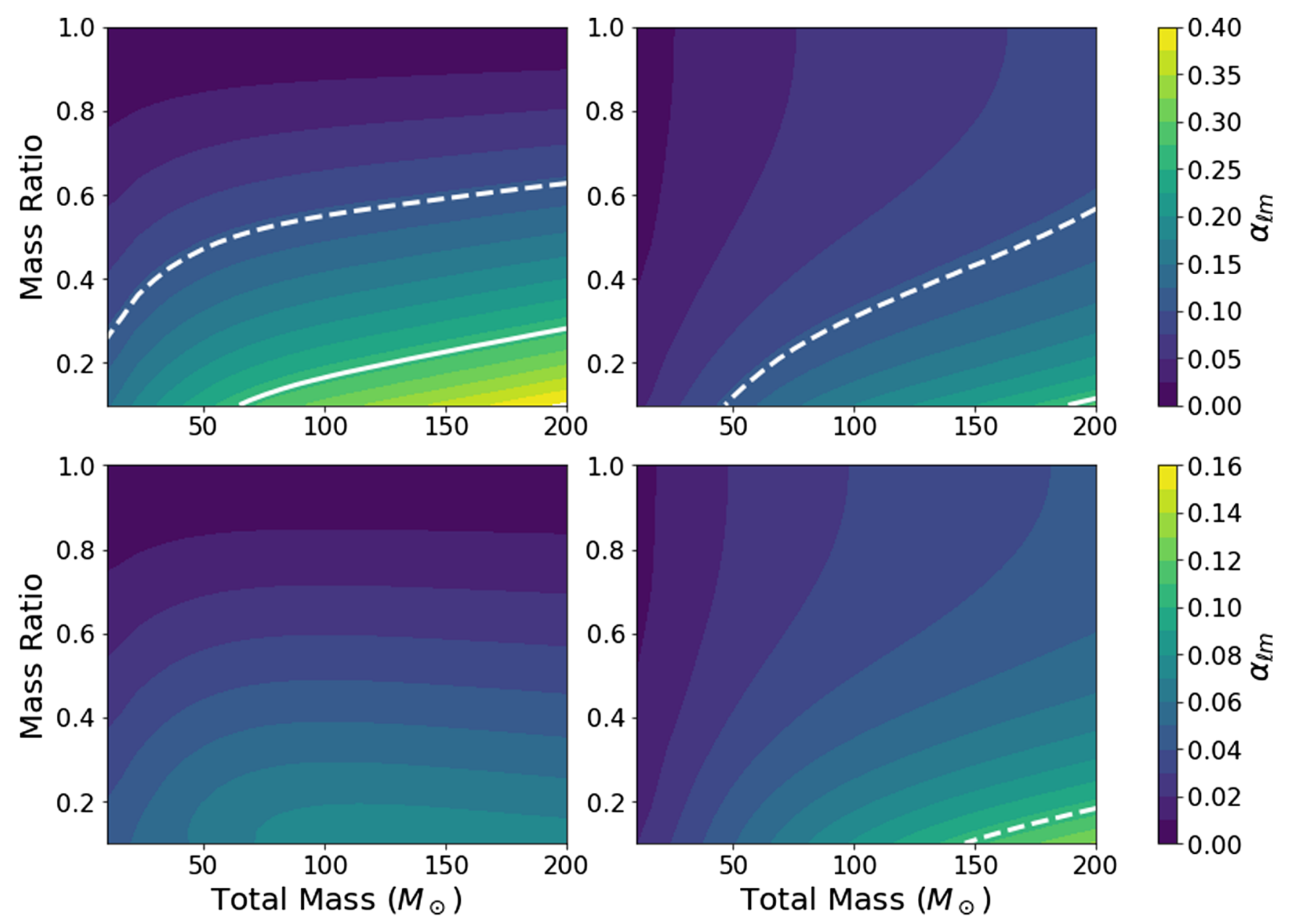

FIG. 2. Ratio of the intrinsic amplitude, $\alpha_{l m}$, [defined in Eq. (13)] of signal harmonics to the $(2,2)$ harmonic as a function of the total (detector frame) mass and mass ratio of the system, in a detector with sensitivity matching the Advanced LIGO detectors during O3 [67]. Upper left: the $(3,3)$ harmonic; Upper right: the $(4,4)$ harmonic; lower left: the $(2,1)$ harmonic; lower right: the $(3,2)$ harmonic. In all cases, the spins of the black holes are set to zero. The solid white line corresponds to $\alpha_{\ell m}=5.3 / 20$ and the dashed line to $\alpha_{\ell m}=2.1 / 20$, which correspond, approximately, to the threshold for the higher harmonics being confidently/marginally observable for a signal with $\mathrm{SNR}=20$ in the $(2,2)$ multipole. Note that the color bar is normalized differently between the top and bottom row to improve the visibility of the weaker harmonics.

$(2,1)$ and $(3,2)$ multipoles can be significant. These overlaps are shown in Fig. 3 as a function of total mass and mass ratio. As the $(2,1)$ multipole has approximately the same frequency as the $(2,2)$ multipole during ringdown, we expect a significant overlap at higher masses when the (merger and) ringdown occur within the sensitive band of the detector. Similarly, for the $(3,2)$ multipole, the frequency evolution during the inspiral matches closely with the $(2,2)$ multipole and we therefore expect a significant overlap between the $(2,2)$ and $(3,2)$ multipoles, particularly for low masses. Consequently, it can be difficult to identify these harmonics in the signal. Interestingly, one of the most significant impacts of the $(3,2)$ multipole can be to produce an incorrect estimate of the amplitude of the $(2,2)$ harmonic, and consequently introduce an error in the measured distance, as power from the $(3,2)$ multipole will be mistakenly attributed to the $(2,2)$ harmonic [45].

\section{B. Dependence upon extrinsic parameters}

The observed SNR in the higher harmonics depends upon the orientation of the binary, through the $R_{\ell m}$ factor defined in Eq. (14), in addition to the intrinsic amplitude of the harmonics discussed above. We can make several immediate observations from Fig. 1 or, equivalently, directly from the functional form of the spin-weighted spherical harmonics. The $(3,3),(4,4)$ and $(2,1)$ multipoles vanish for a signal observed face-on $(l=0)$, so the minimum value of $R_{\ell m}$ for these harmonics is zero; in contrast, there is no orientation for which both polarizations of the $(3,2)$ harmonic vanishes. Next, there is no orientation where the $(2,2)$ harmonic vanishes, but the other harmonics do not-the $(2,2)$ harmonic only vanishes for the $\times$ polarization for an edge-on system, but all other harmonics we are considering also vanish there. Thus, there is a finite, maximum value of $R_{\ell m}$ for all harmonics, and it's 

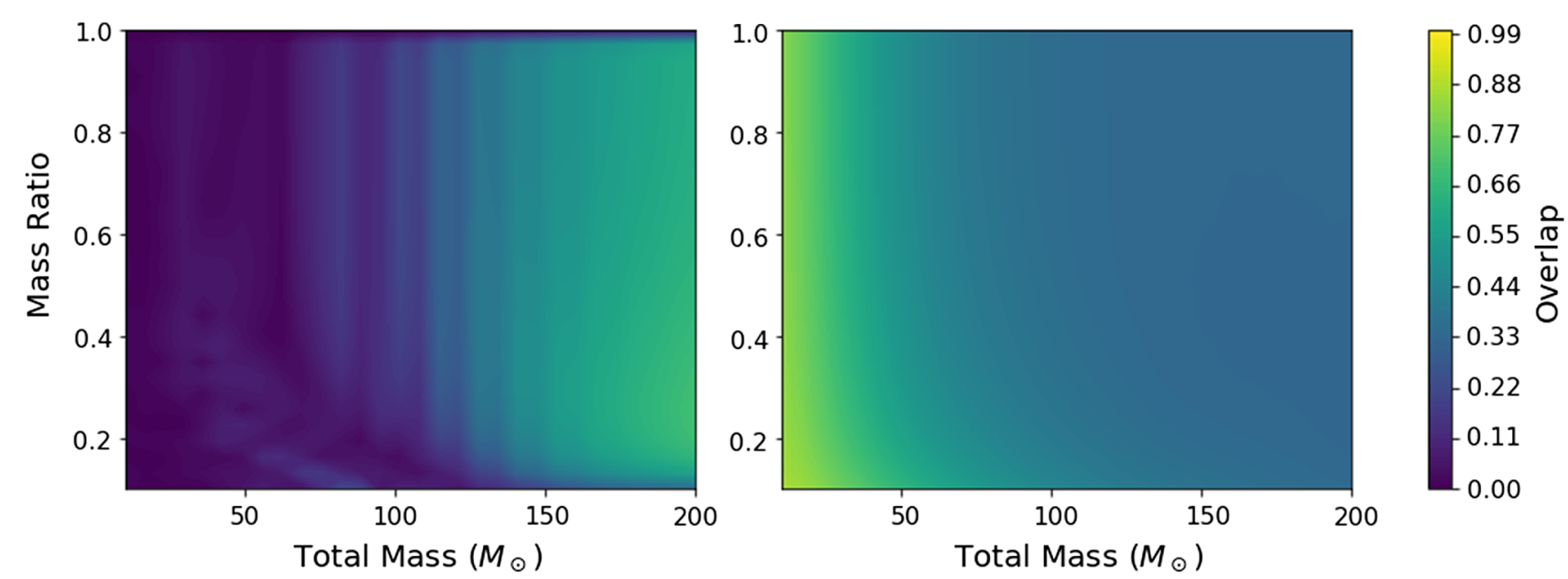

FIG. 3. Absolute value of the noise-weighted inner product between multipoles, evaluated using the Advanced LIGO (O3) sensitivity, as a function of mass ratio and total mass for non-spinning black holes $\left(\chi_{\text {eff }}=0\right)$. Left: the overlap between the $(2,2)$ and $(2,1)$ multipoles; Right: the overlap between $(2,2)$ and $(3,2)$ multipoles.
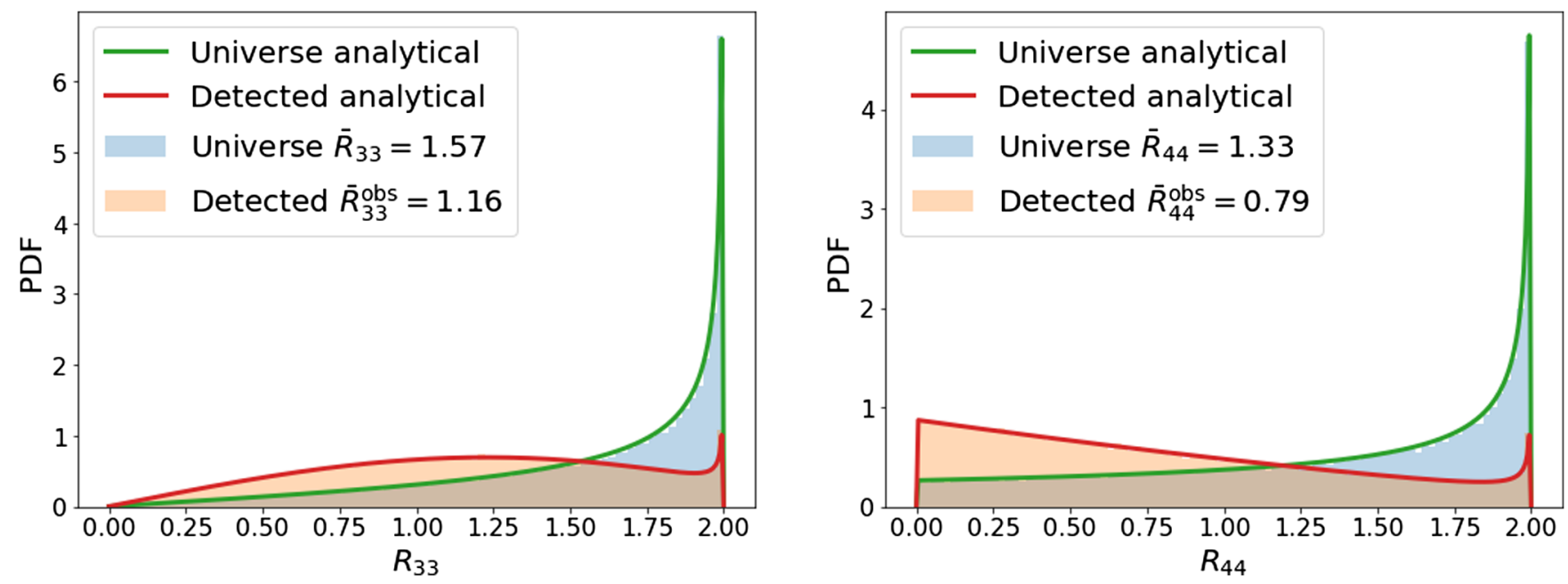

FIG. 4. Distribution of $R_{33}(l)$ and $R_{44}(l)$ for all binaries (Universe) as well as that subset that would be detected above a fixed SNR threshold for the $(2,2)$ harmonic (Detected). We show both the results from a Monte-Carlo simulation as well as the analytical prediction.

easy to see from Fig. 1 that $R_{\ell m}^{\max }=2$, which occurs for edge-on systems.

In Fig. 4 we plot the distribution of the geometrical factors $R_{33}(l)$ and $R_{44}(l)$. We have restricted attention to the $(3,3)$ and $(4,4)$ multipoles as these are the most significant, as seen in Fig. 2, and also the expression for $R_{\ell m}$ is independent of the observed gravitational wave polarization. We consider the distribution of $R_{\ell m}$ for a population of sources distributed uniformly in volume $5^{5}$ and with uniformly distributed orientation. We show both the

\footnotetext{
${ }^{5}$ Realistically, we do not expect sources to be uniformly distributed, due to both cosmological effects and a redshift dependent star formation and, hence, merger rate [68]. Nonetheless, this simple model provides a reasonably approximation to gain an understanding of the likely values of $R$.
}

distribution of $R_{\ell m}$ for a uniform population of sources, as well as the expected observed distribution. In order to obtain the observed distribution for $R_{33}(l)$ and $R_{44}(l)$, it is sufficient to consider selection effects only on the inclination angle. If binaries are uniformly distributed in volume and orientation the distribution of inclinations for signals above an arbritrary detection threshold is known analytically [69] [see Eq. (B3)]. This allows us to derive analytical expressions for the $p\left(R_{33}(l)\right)$ and $p\left(R_{44}(l)\right)$ distributions shown in Fig. 4, which we do in Appendix B.

For both the $(3,3)$ and $(4,4)$ harmonics, the distribution peaks at $R_{\ell m}=2$, the value for an edge-on system, with mean values of $\bar{R}_{33}=1.57$ and $\bar{R}_{44}=1.33$. However, since the emission in the $(2,2)$ harmonic is weakest when the system is observed edge on, selection effects serve to significantly reduce the peak in the observed population. 
For the $(3,3)$ harmonic, the peak remains at $R_{33}^{\text {obs }}=2$, but the distribution is broad, with significant support over the full range from 0 to 2 and a mean value of $\bar{R}_{33}^{\text {obs }}=1.16$. The mode of the observed $R_{44}^{\text {obs }}$ distribution is zero, although again there is broad support over the range from 0 to 2 with a mean value of $\bar{R}_{33}^{\text {obs }}=0.79$.

For other harmonics, the expected distribution of $R_{\ell m}$ will depend upon the sensitivity of the detector network to the two polarizations of the gravitational wave-the distribution for $R_{\ell m}$ will differ between a single detector, sensitive to only one polarization, and a network with good sensitivity to both polarizations. Nonetheless, the distribution for $R_{21}$ will share features with $R_{33}$ and $R_{44}$, namely it will take values between 0 (face on) and 2 (edge on), with a peak at $R_{21}=2$ which is reduced by selection effects in the observed population. The distribution for $R_{32}$ will also be bounded between 0 and 2, although, due to the fact that the $(3,2)$ multipole does not vanish for face-on sources, there is also a significant contribution at $R_{32} \approx 1$ from face-on sources.

\section{OBSERVING HIGHER HARMONICS}

When a gravitational wave signal from a binary merger is observed, it is natural to ask whether the higher multipoles have been observed. Typically, the searches that identify events do not use higher harmonics to extract events from the data [70-72] (although see [23] for ways to incorporate them). However, parameter estimation routines do incorporate higher harmonics into the recovery of parameters, and a natural way to ask whether higher harmonics have been observed is to calculate the Bayes factor (or odds ratio) between parameter recovery with and without higher multipoles in the waveform $[42,43]$. In this section, we show that the SNR in higher harmonics is also an effective tool in determining observability of higher harmonics.

\section{A. Measured SNR in higher harmonics}

We assume that the $(2,2)$ harmonic has been observed and consider the SNR contained in the higher multipoles. As in Eq. (7), we consider only two harmonics, the $(2,2)$ harmonic and a single additional harmonic. Since the $(2,2)$ harmonic has been identified, it is straightforward to calculate the SNR in the $(\ell, m)$ harmonic, by generating the $\tilde{h}_{\ell m}$ waveform, with the same masses, spins and arrival time, and filtering it against the data. If the overlap between the $(\ell, m)$ and $(2,2)$ harmonics is nonzero, then this will pick up power contained in the $(2,2)$ harmonic, and it is necessary to remove it by first computing the orthogonal component,

$\tilde{h}_{\ell m}^{\perp}=\tilde{h}_{\ell m}-\frac{1}{\left|\tilde{h}_{22}\right|^{2}}\left[\left(\tilde{h}_{\ell m} \mid \tilde{h}_{22}\right) \tilde{h}_{22}+\left(\tilde{h}_{\ell m} \mid i \tilde{h}_{22}\right) i \tilde{h}_{22}\right]$.

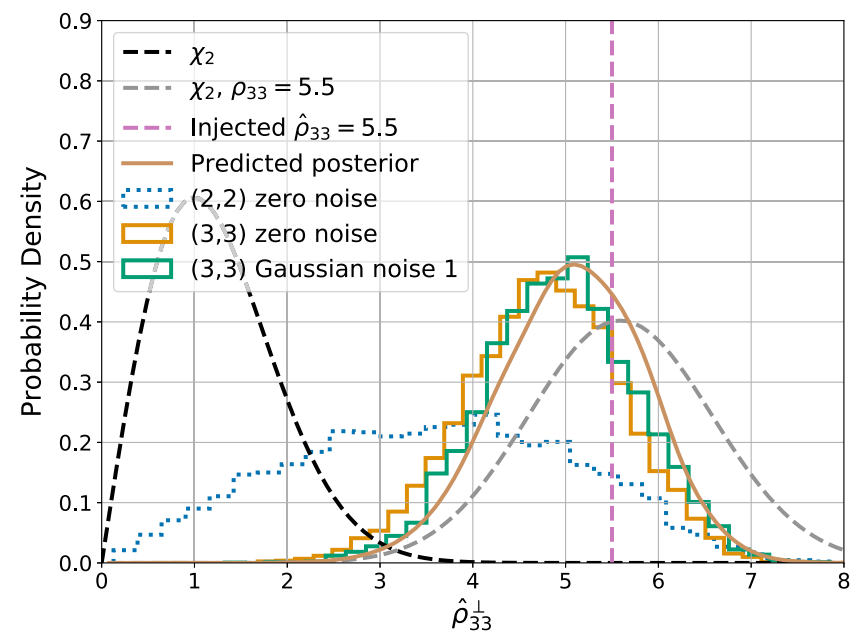

FIG. 5. Posterior probability distribution for $\hat{\rho}_{33}^{\perp}$, the orthogonal optimal signal-to-noise ratio of the $(3,3)$ multipole. The simulated waveform corresponds to system with $m_{1}=40 M_{\odot}$, $m_{2}=10 M_{\odot}$ and $\cos \imath=0.7$. The two solid histograms show the posterior distribution when the $(3,3)$ multipole is included in the waveform, either with (green) or without (orange) Gaussian noise. The dotted histogram shows the posterior on the SNR in the $(3,3)$ harmonic inferred from the measurement of the $(2,2)$ harmonic alone. The vertical line indicates the simulated value of $\hat{\rho}_{33}^{\perp}$ and the dashed lines indicate the expected distribution, based on a non-central $\chi$ distribution with two degrees of freedom and assuming a flat prior (as derived in Sec. IV B), in the presence and absence of a signal in the $(3,3)$ harmonic. Also shown as a solid brown line is the predicted posterior distribution for a signal with $\hat{\rho}_{33}^{\perp}=5.5$, but instead using the inferred distribution from the measurement of the $(2,2)$ harmonic (the dotted blue histogram) as a prior. We see general agreement between the predicted and measured posteriors, with the measured values slightly smaller than predicted (see Sec. IV C for discussion).

Here, $\tilde{h}_{22}$ and $i \tilde{h}_{22}$ denote the two orthogonal phases of the $(2,2)$ harmonic. Filtering $\tilde{h}_{\ell m}^{\perp}$ against the data, $s$, gives

$$
\left(\rho_{\ell m}^{\perp}\right)^{2}=\frac{1}{\left|\tilde{h}_{\ell m}^{\perp}\right|^{2}}\left[\left(s \mid \tilde{h}_{\ell m}^{\perp}\right)^{2}+\left(s \mid i \tilde{h}_{\ell m}^{\perp}\right)^{2}\right] .
$$

When the parameters of the waveform are known, or have been inferred through parameter estimation, we can calculate the expected SNR in the $(\ell, m)$ multipole as

$$
\hat{\rho}_{\ell m}^{\perp}=\hat{\rho}_{\ell m} \sqrt{1-O(\ell m, 22)^{2}},
$$

where $\hat{\rho}_{\ell m}$ is the expected SNR in the $(\ell, m)$ harmonic, as defined in Eq. (12) and $O(\ell m, 22)$ is the overlap between the $(\ell, m)$ and $(2,2)$ waveforms as defined in Eq. (16).

In Fig. 5 we show the inferred posterior probability distribution for $\hat{\rho}_{33}^{\perp}$ for a binary with masses $m_{1}=40 M_{\odot}$, $m_{2}=10 M_{\odot}$ inclined at $\cos l=0.7\left(l \approx 45^{\circ}\right)$ and with $\rho_{22}=22$ under a variety of assumptions for signal and 

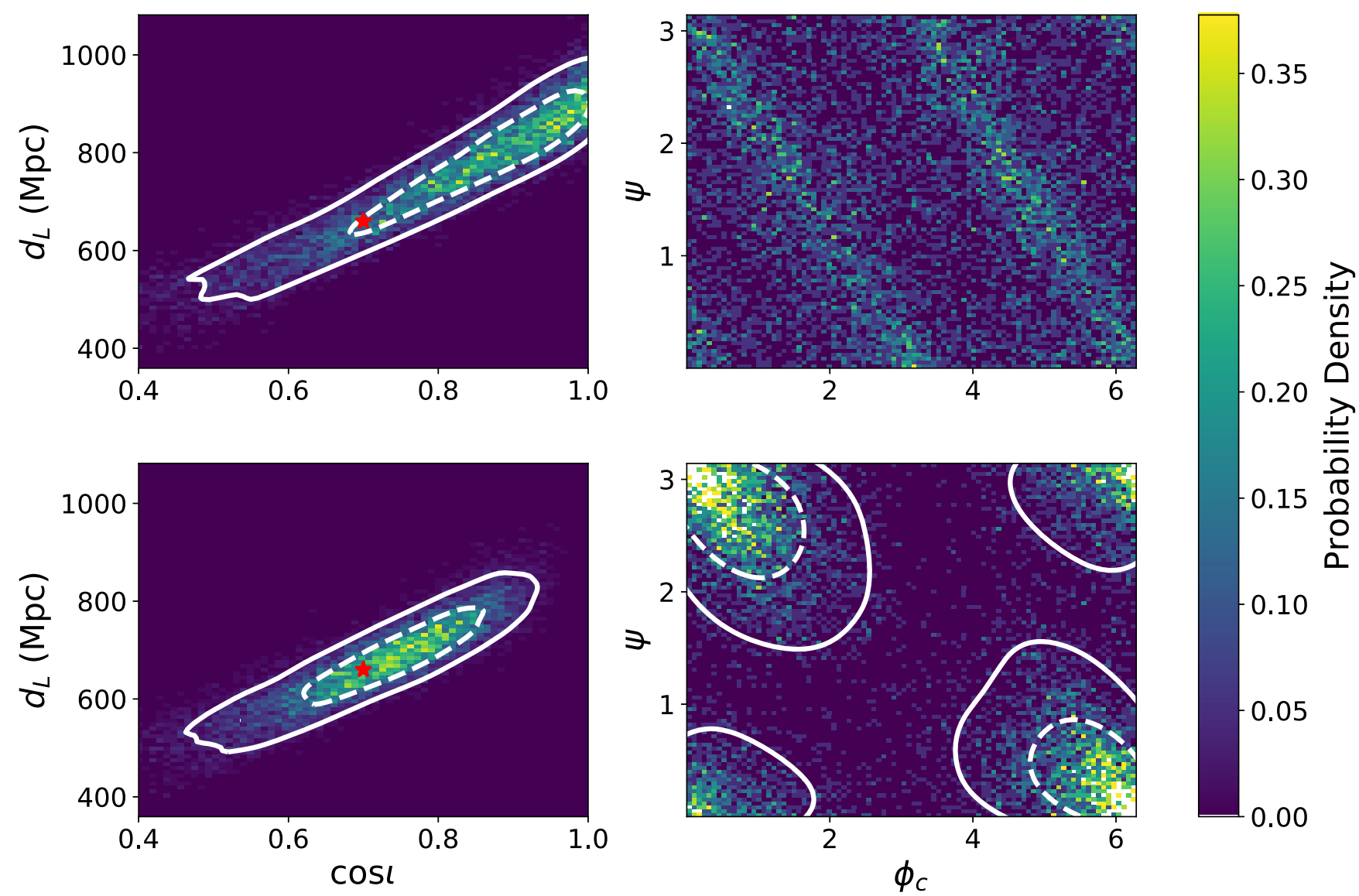

FIG. 6. 2D Posterior probability distribution for left: inclination and distance, right: polarization and phase at coalescence for a signal model containing top: only the dominant $(2,2)$ multipole and bottom: the $(2,2)$ and $(3,3)$ multipoles. The simulated waveform corresponds to a system with $m_{1}=40 M_{\odot}, m_{2}=10 M_{\odot}$ and $\cos \imath=0.7$. The solid (dashed) white contours denote $90 \%$ (50\%) credible regions. These are not shown for polarization-phase for the $(2,2)$ waveform, due to the clear degeneracy.

model. ${ }^{6}$ For the simulated signal the relative amplitude of the $(3,3)$ harmonic is $\alpha_{33} \approx 0.18$, and the orientation factor is $R_{33}=1.4$, which implies $\hat{\rho}_{33} \approx 5.5$ and, since the overlap between $(2,2)$ and $(3,3)$ harmonics is small, $\hat{\rho}_{33}^{\perp} \approx \hat{\rho}_{33}$. The recovered distribution of $\hat{\rho}_{33}^{\perp}$, both with and without noise $[(3,3)$ zero noise and $(3,3)$ Gaussian noise in the figure], matches well with the simulated value but is shifted to lower values. We discuss this shift in Section IV C.

We can also infer the power in the $(3,3)$ harmonic even when we use only the $(2,2)$ harmonic to recover the parameters, particularly masses and binary orientation, of the system. Unsurprisingly, the distribution of $\hat{\rho}_{33}^{\perp}$ no longer matches well with the simulated value and now spreads over a broad range from 0 to 8 . In this case, it seems clear that the $(3,3)$ harmonic has been observed, as its inclusion leads to a significant change in the inferred SNR in the $(3,3)$ harmonic.

\footnotetext{
${ }^{6}$ All parameter estimates reported in this paper were obtained with LALInference [73] assuming a HLV network with the sensitivities achieved during O3 [67].
}

In Fig. 6 we show the inferred posterior probability distributions of inclination, distance, polarization and phase at coalescence using waveform models that do/do not include the higher harmonics. Although the binary is generated with the orbital plane inclined at an angle of $l \approx 45^{\circ}$, using only the $(2,2)$ harmonic, the system is recovered consistent with face-on, due to well-known degeneracies between distance in inclination [48]. Consequently, the only well measured quantities are the amplitude and phase of the circularly polarized waveform that is recovered: $A_{22} \approx \frac{\cos l}{D_{L}}$ and $\phi_{22} \approx \psi+\phi_{o}$, with the inclination bounded between $0 \leq \imath \leq 45^{\circ}$. When the $(3,3)$ harmonic is added, the degeneracy is broken and the distance, inclination, polarization and phase are all measured with good accuracy.

\section{B. Expectation due to noise}

The question, then, is whether an observed SNR in a given higher harmonic is evidence that the higher harmonic has been observed, or if this is to be expected due to noise alone. Similar questions have been addressed multiple 
times in the gravitational wave literature, for example in [74-78]. Here, we follow the methods developed in those earlier papers, focusing on a specific application to higher harmonics. We calculate the expected distribution of $\hat{\rho}_{\ell m}^{\perp}$ under some simplifying assumptions. Specifically, we consider the scenario where measurement of the $(2,2)$ harmonic has already fixed the parameters which determine the phase evolution of the binary (primarily the chirp mass, but also a combination of aligned spin and mass ratio [78]), the time of arrival and sky location of the system. Furthermore, we assume that the $(\ell, m)$ multipole is the second most significant (in many cases, this is the $(3,3)$ harmonic), and other multipoles do not contribute significantly.

We will treat the overall amplitude and phase of the $(\ell, m)$ multipole as unconstrained by the observation of the $(2,2)$ harmonic. As shown in Fig. 6, there are degeneracies in the measurement of distance/inclination and polarization/phase when observing only the $(2,2)$ harmonic. The amplitude of the higher harmonics, and in particular the $(3,3)$ and $(4,4)$ harmonics, varies significantly over the range $0^{\circ} \leq \imath \leq 45^{\circ}$ and can therefore be treated as unconstrained. Similarly, the overall phase of these multipoles differs from the $(2,2)$ by a factor of $(m-2) \phi_{o}$ and is therefore unconstrained by the measurement of the $(2,2)$ harmonic. Another way to see this is to look at the posterior probability distribution for the $(3,3)$ amplitude inferred when using a waveform containing only the $(2,2)$ multipole in Fig. 5. The distribution is broad and has support across a large range of $\hat{\rho}_{33}^{\perp}$. This argument will only hold for the subdominant harmonic: once the amplitude of a second harmonic is fixed, the four orientation parameters of the binary are determined and, consequently, the amplitude of the remaining harmonics is significantly constrained.

We are interested in obtaining the expected distribution for $\hat{\rho}_{\ell m}{ }^{7}$ under the noise-only hypothesis (i.e., only power in the $(2,2)$ harmonic). In this case, we are fitting the data with a template waveform

$$
\tilde{h}=a \tilde{h}_{\ell m}+i b \tilde{h}_{\ell m}+c \tilde{h}_{22}+i d \tilde{h}_{22},
$$

where $\tilde{h}_{\ell m}$ and $i \tilde{h}_{\ell m}$ are the two phases of the waveform of the $\ell m$ harmonic, $a$ and $b$ control the overall amplitude of this harmonic, and $\tilde{h}_{22}, c$ and $d$ give the contribution of the dominant harmonic to the waveform. We are interested in the expected distribution of $a$ and $b$ when there is no power in higher harmonics. Based upon the discussion above, we choose a uniform prior $\pi(a, b)$ on $a$ and $b$. In what follows we neglect the terms related to the dominant harmonic as they are unaffected, to the level of our approximation, by the presence of the higher harmonics. The posterior will be

\footnotetext{
${ }^{7}$ For simplicity of presentation, we drop the ${ }^{\perp}$ from the equations in the remainder of the section. Where the harmonic has overlap with the $(2,2)$ waveform, the calculation should be understood to be performed with the orthogonal component.
}

$$
p(a, b \mid s) \propto \Lambda(a, b) \pi(a, b) .
$$

where the likelihood of a signal s given the amplitudes $a, b$ and Gaussian noise is

$$
\Lambda(s \mid a, b) \propto \exp \left[-\frac{1}{2}(s-\tilde{h}(a, b) \mid s-\tilde{h}(a, b))\right] .
$$

Using polar variables $\hat{\rho}_{\ell m}=\sqrt{a^{2}+b^{2}}$ and $\hat{\phi}_{\ell m}=$ $\arctan (b / a)$, and assuming a uniform prior we can write the posterior probability distribution for the amplitudes $a$ and $b$ given a signal $\mathrm{s}$ as

$$
\begin{aligned}
& p(a, b \mid s) d a d b \propto \Lambda(a, b) d a d b \\
& \propto e^{\left[a\left(s \mid h_{\ell m}\right)+b\left(s \mid i h_{\ell m}\right)-\frac{a^{2}+b^{2}}{2}\right]} d a d b \\
& =\hat{\rho}_{\ell m} d \hat{\rho}_{\ell m} d \hat{\phi}_{\ell m} \\
& \quad \times e^{\left[-\frac{\hat{\rho}_{\ell m}^{2}}{2}+\hat{\rho}_{\ell m}\left[\cos \hat{\phi}_{\ell m}\left(s \mid \tilde{h}_{\ell m}\right)+\sin \hat{\phi}_{\ell m}\left(s \mid \tilde{h}_{\ell m}\right)\right]\right]} .
\end{aligned}
$$

Defining the matched filter signal-to-noise ratio, $\rho_{\ell m}$ as in Eq. (18) and the phase

$$
\phi_{\ell m}=\arctan \frac{\left(s \mid i \tilde{h}_{\ell m}\right)}{\left(s \mid \tilde{h}_{\ell m}\right)}
$$

and marginalizing over $\hat{\phi}_{\ell m}$, we obtain

$$
\begin{aligned}
p\left(\hat{\rho}_{\ell m} \mid s\right) & \propto \hat{\rho}_{\ell m} e^{\left[-\frac{\left.\hat{\rho}_{\ell m}^{2}\right]}{2}\right.} \int_{0}^{2 \pi} e^{\left[\hat{\rho}_{\ell m} \rho_{\ell m} \cos \left(\hat{\phi}_{\ell m}-\phi_{\ell m}\right)\right]} d \hat{\phi}_{\ell m} \\
& \propto \hat{\rho}_{\ell m} \exp \left[-\frac{\hat{\rho}_{\ell m}^{2}+\rho_{\ell m}^{2}}{2}\right] I_{0}\left(\hat{\rho}_{\ell m} \rho_{\ell m}\right)
\end{aligned}
$$

where $I_{0}$ is the modified Bessel function of the first kind. We recognize Eq. (24) as the noncentral chi distribution with 2 degrees of freedom and noncentrality parameter equal to $\rho_{\ell m}$. In the absence of signal power in the higher harmonics, the probability distributions for the filters $\left(s \mid \tilde{h}_{\ell m}\right)$ and $\left(s \mid i \tilde{h}_{\ell m}\right)$ are zero-mean, unit-variance Gaussians and $\rho_{\ell m}$ is chi-distributed with 2 degrees of freedom.

\section{Observation of higher harmonics}

In Fig. 7 we show the distribution of $\hat{\rho}_{33}$, in the absence of a signal in the $(3,3)$ harmonic. First, we have the recovered distribution when performing parameter estimation on a signal containing only the $(2,2)$ harmonic and the zero instance of the noise distribution. Based upon the calculation above, we expect this to follow the $\chi$ distribution with two degrees of freedom, and we see that it does. We also show the distribution for three different instances of Gaussian noise. In each of these cases, the 


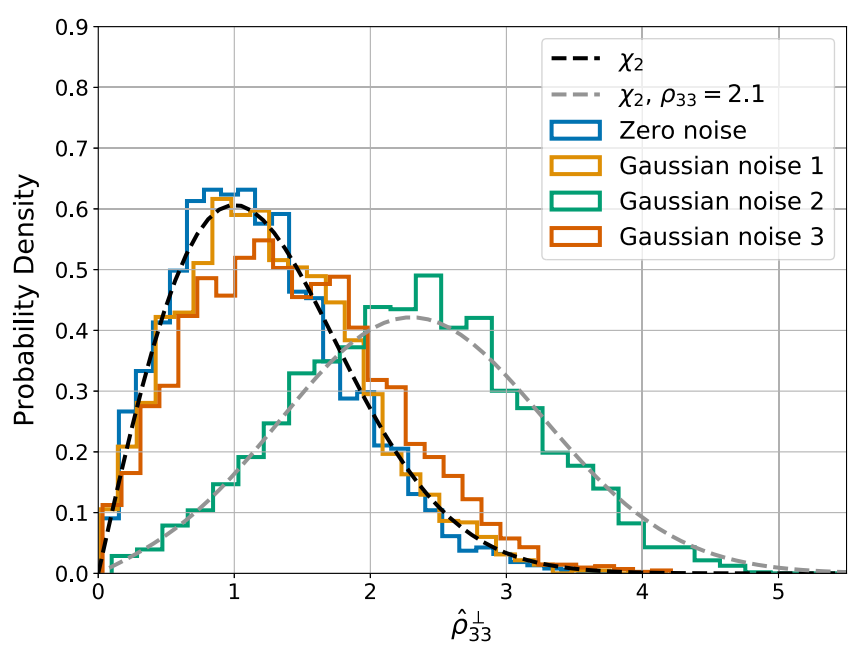

FIG. 7. Posterior probability distribution for $\hat{\rho}_{33}^{\perp}$, the orthogonal optimal signal-to-noise ratio of the $(3,3)$ multipole, when the simulated signal contains the $(3,3)$ multipole for a variety of models and noise realizations. The injected parameters are $m_{1}=40 M_{\odot}, m_{2}=10 M_{\odot}$ at $\cos \imath=0.7$.

distribution is expected to follow a noncentral $\chi$ distribution, where the noncentrality parameter is given by the matched filter SNR in the $(3,3)$ harmonic-in this case, there is no signal and any power is simply due to noise. For two of the noise realizations (Gaussian noise 1 and Gaussian noise 3$)$, there was minimal power in the $(3,3)$ harmonic and the $\hat{\rho}_{33}$ distribution matches closely with the zero-noise case. However, in the Gaussian noise 2 realization, the SNR in the $(3,3)$ multipole is higher, and the mode of the distribution is moved significantly away from zero.

We propose a simple test for the observability of higher multipole moments in a gravitational waveform: if the SNR in the second most significant harmonic satisfies $\rho_{\ell m}>2.1$, this signifies the observation of power in the higher multipole moments. We have argued that the matched filter SNR, in the absence of signal, will be well approximated by a $\chi$ distribution with two degrees of freedom. We expect Gaussian noise to produce an SNR greater than 2.1 less than $10 \%$ of the time and therefore require $\rho_{\ell m}>2.1 .^{8}$

The estimate of $\rho_{\ell m}$ can be obtained either by matched filtering, or by fitting the measured distribution of $\hat{\rho}_{\ell m}$ from parameter estimation results and obtaining the noncentrality parameter. Based on this criteria, our third noise trial would show marginal evidence for presence of the $(3,3)$

\footnotetext{
${ }^{8}$ In [79], the authors propose an observability criterion specifically for higher harmonics observed during the ringdown of the final black hole. They require an SNR in each harmonic above 5, corresponding to our 5-sigma detection. In addition, require distinguishability between the ringdown mode frequencies which (as can be seen in their Table 1) is virtually always satisfied if the modes can be observed.
}

harmonic. This prescription can be easily extended to a criterion for confident detection of the higher harmonics: a "5-sigma" observation could correspond to $\rho_{\ell m}>5.3$. In Fig. 2, we have added contours at values of $\alpha_{\ell m}=2.1 / 20$ and 5.3/20. These indicate the approximate boundaries in the mass space where higher harmonics would be marginally/confidently observed for a signal at SNR = 20. Of course, the actual higher harmonic SNR will depend also on the orientation factor $R_{\ell m}$, which varies between 0 and 2 , with a median value around 1 for the $(3,3)$ and $(4,4)$ harmonics.

Next, we return to Fig. 5 and note that the distribution of $\hat{\rho}_{33}$ for the signal containing higher harmonics matches well with expectation-a noncentral $\chi$ distribution with non centrality parameter 5.5-but is shifted to lower values. A more accurate prediction for the distribution can be made by revisiting our assumptions. The derivation of the expected distribution Eq. (24), assumed a uniform prior for $\hat{\rho}_{33}$. We note that the dotted histogram in Fig. 5 is the probability of $\hat{\rho}_{33}$ before we probe the likelihood associated with the $(3,3)$ harmonic and so is approximately equivalent to the prior on $\hat{\rho}_{33}$. Thus, the prior distribution is broad but not flat. The solid curve in Fig. 5 shows the product of the likelihood in Eq. (24) with the prior as approximated by the dotted histogram. We see that this results in a more accurate predicted posterior. Remaining differences between the predicted and measured posteriors can be sourced to our assumption that the phase evolution (or equivalently the masses and spins) of the waveform templates are fixed. During inference, as the masses and spins are varied, the matched filter SNR is never larger and is usually lower than the simulated value. By fixing the SNR to the simulated value we therefore overestimate the actual value and the distribution is shifted to larger values of $\hat{\rho}_{33}$.

An alternative method of establishing the observability of higher harmonics is to compare the Bayes factor (or evidence) between a waveform model additionally containing the $(3,3)$ multipole and a model with only the $(2,2)$ multipole $[42,43]$. The difference in Bayes factor, obtained by marginalizing the likelihood [73], between the two parameter estimation runs (with and without $(3,3)$ harmonic) is $\log _{10} \mathrm{BF}=4.7$. We can compare this to our results on the SNR in the higher harmonics by noting that an increase in SNR corresponds to an increase of the likelihood by a factor of $\approx e^{\rho^{2} / 2}$. Our injected value of $\rho_{33}=5.5$, leads to an estimate of $\log _{10}$ Bayes factor of 6.6 ( $\log _{10}$ of the increase in the likelihood). For a more accurate comparison, we should also account for the prior distribution, as well as the width of the posteriors. Since both the $(2,2)$ only and higher harmonic waveforms are described by the same parameters, the priors are unchanged. However, as is clear from Fig. 6, the posterior is significantly more peaked when the higher harmonics are included. The improved constraints from the $(3,3)$ 
multipole reduce the prior volume by a factor of $\sim 2$ in the distance inclination plane (assuming a uniform in volume prior), and a factor of $\sim 5$ in the polarization phase plane. This implies the Bayes factor based purely on the increased likelihood be reduced by a factor of $\sim 10$, equivalent to reducing the $\log _{10}$ Bayes factor by one to 5.6. Finally, as discussed above, the higher harmonic SNR is generally inferred to be smaller than the simulated value. Indeed, from Fig. 5, the peak occurs at $\hat{\rho}_{33} \sim 5.0$, which corresponds to a $\log _{10}$ Bayes factor of 4.4. This is in close, but not perfect agreement with the full parameter estimation result.

\section{HIGHER HARMONICS IN A POPULATION OF BINARY MERGERS}

Here, we consider the likelihood of observing the higher harmonics in signals drawn from a population. To do so, we generate a large number of potential signals from a given population and assess which would be observable above a given threshold and, of those, which would have sufficient power in the $(3,3)$ and/or $(4,4)$ harmonics for them to be observable (above the threshold of $\rho_{\ell m}=2.1$ ). We choose a mass distribution of black holes in binary systems where the mass of the more massive black hole is taken from a power-law distribution $p\left(m_{1}\right) \propto m_{1}^{-\alpha}$ and choose the power law parameter of $\alpha=-2.35$, while restricting the mass to lie in the range $[5,50] \mathrm{M}_{\odot}$; the distribution for $m_{2}$ is taken to be uniform in the range $\left[5 M_{\odot}, m_{1}\right]$. The spins of the individual black holes are assumed to be isotropically distributed, with low spin magnitudes (the magnitude is a triangular distribution peaked at spin magnitude of zero and falling to zero at maximal spin) [80]. Binaries are assumed to be isotropic on the sky, with uniform orientations and distances distributed uniformly in comoving volume.
In Fig. 8 we show the subset of this population which would be detectable with the HLV network operating at the sensitivities achieved during $\mathrm{O} 3$ [67]. More pertinently, we also plot the subset from this detected population which result in gravitational waves with a measurable signal in the two loudest subdominant multipoles. Overall around $5.5 \%$ of binaries are expected to have sufficient power in the higher harmonics for them to be observed. Of these, the vast majority will have an observable $(3,3)$ harmonic $(5.3 \%)$, with the $(4,4)$ harmonic observable in $0.85 \%$ of binaries, but for the majority of these, the $(3,3)$ harmonic will also be observable. Only two to three observable events in 1,000 from this population are expected to have an observable $(4,4)$ harmonic but not observable $(3,3)$.

The higher harmonics are preferentially observable in signals with unequal masses and for binaries which are significantly inclined. In particular, for binaries with mass ratio between $4: 1$ and 10:1, the majority of signals will have observable higher harmonics, and even at a mass ratio of $2: 1$, around $10 \%$ of binaries will have observable higher harmonics. Convolving the observed distribution with the fraction of binaries with significant higher harmonics gives a peak of signals with observable higher harmonics around a mass ratio of $3: 1$. Interestingly, for binaries close to equal mass, it is the $(4,4)$ harmonic which is more likely to be observed, and essentially all binaries where the $(4,4)$ but not $(3,3)$ is observed have close to equal masses (between $1: 1$ and $5: 4)$.

\section{DISCUSSION}

We have explored the relative significance of the higher gravitational wave harmonics in binary merger signals. For simplicity, we have decomposed the harmonics into an overall amplitude-dependent upon the masses and spins
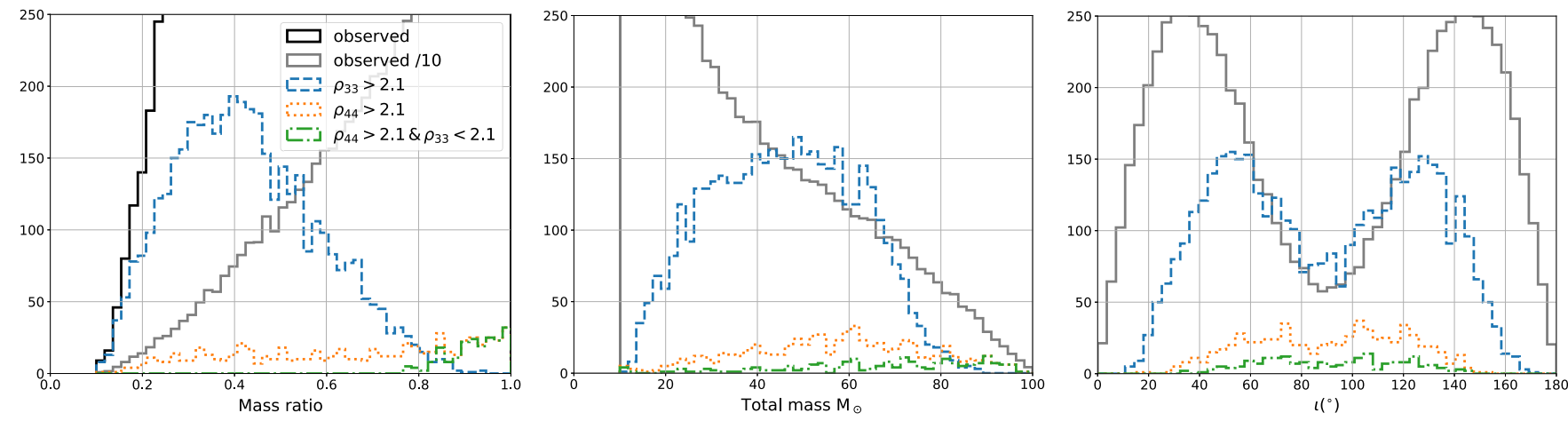

FIG. 8. The mass ratio, total mass and orientation distribution for a simulated population of black hole binaries, and for that subset of systems for which the $(3,3)$ or $(4,4)$ harmonics are visible. The population is modeled as a power law in masses, with isotropic distribution of orientations, further details of the population are given in the text. The distribution is shown as a function of Left: mass ratio; Middle: total mass; Right: orientation. The subset of sources for which the $(3,3)$ or $(4,4)$ multipole moment is observable are shown by the dashed/dotted lines respectively, and those with observable $(4,4)$ multipole where the $(3,3)$ multipole is not observable are shown by the dot-dashed line. We show the observed population divided by 10 as a grey solid line, and on the mass ratio plot, without reweighting as a black line. The total number of observed binaries in the simulation is 78,000. 
of the system-and an orientation-dependent termdependent upon the inclination and polarization. This allows us to easily identify the most significant harmonics, and the regions of parameter space where they are most likely to be observable. As is well known $[9,10,47]$, the higher harmonics are most significant when the binary is observed edge on. As expected, our orientation amplitudes are largest for edge-on systems although, due to selection effects, we observe that the most likely observed configuration is a binary with axes orientated at around $45^{\circ}$ to the line of sight. In addition, we show that for much of the binary parameter space, the $(3,3)$ multipole will be the most significant subdominant harmonic, with an amplitude about one tenth of the $(2,2)$ harmonic for a massratio 2 binary (over a broad range of masses). The $(4,4)$ multipole becomes more significant at higher masses and, although the relative amplitude is less than 0.1 for much of the parameter space, it is still the most significant subdominant harmonic for high-mass systems where the two components have comparable masses.

For signals which are observed at low SNR, it is likely that at most one additional harmonic will be clearly observable. Thus, for simplicity, we have introduced an observability criterion for the second harmonic. In many cases, the amplitude and phase of the second harmonic is largely unconstrained by the observation of the $(2,2)$ multipole: there are often large degeneracies between the measurement of the distance and inclination of the binary and also the polarization and phase [48]. Consequently, in the absence of a signal, the power in the second most significant harmonic will be $\chi^{2}$ distributed with two degrees of freedom, corresponding to the unknown amplitude and phase of the harmonic. If there is power in the higher harmonic, the distribution will be noncentral $\chi^{2}$, where the noncentrality is given by the SNR in the higher harmonic. We have performed a series of simulations that demonstrate this expectation is valid. Using this simple observation, we have introduced a criterion for observation of power in a higher harmonic: if the observed SNR in the second most significant harmonic is above 2.1, then this is unlikely to occur due to noise alone so there is marginal evidence of a higher multipole signal, while an SNR $>5.3$ would provide strong ("5-sigma") evidence.

We have identified regions in the parameter space where higher harmonics are most likely to be observed. These regions are those where higher harmonics are likely to be observed, but also which are relatively common in the underlying population of observed gravitational wave signals [46]. We find that these correspond to signals with mass ratios between $2: 1$ and $5: 1$ - for more equal masses, the higher harmonics are too weak, more unequal mass binaries are thought to be rarer. Furthermore, the most likely orientation is for the axis to be inclined at between $30^{\circ}$ and $60^{\circ}$ to the observer-less inclined systems have insufficient power in the higher harmonics while more inclined systems have a weaker overall emission.

There are several applications of the work presented here. As already mentioned, the criterion for observability of higher harmonics has been in used, along with other methods [66], in establishing the presence of power in the $(3,3)$ harmonic in the observed signals GW190412 and GW190814 $[42,43]$. Furthermore, the method can be used in a straightforward way to determine whether it is likely that the higher harmonics will be observable in a given system, and we have provided an example in the population study presented in Sec. V. This is directly applicable to signals observed using a search for the dominant harmonic. Based upon the observed parameters, we can calculate the expected power in the higher harmonics and identify the expected SNR. If significant SNR is expected in higher harmonics, then it becomes worthwhile to undertake the (computationally costly) parameter estimation with waveforms containing several gravitational wave harmonics. This will lead either to the observation of higher harmonics, and the subsequent improvement of parameter measurement, or the nonobservation of higher harmonics and subsequent restriction of the binary parameters to regions of the parameter space where the higher harmonic amplitudes are low.

While the method introduced here is straightforward, there are several clear limitations. Most obviously, the discussion has limited attention to a single observable harmonic. In many cases, this will be a reasonable approximation as there will be one harmonic which is significantly larger than the others (as can be seen from Fig. 2). Furthermore, from simple parameter counting, it seems likely that the observation of a single higher harmonic will be sufficient to significantly improve parameter recovery, most notably the binary orientation. Nonetheless, the observation of additional multipoles will provide additional improvements. For a detailed understanding of the impact of all of the higher harmonics, a full, Bayesian parameter estimation exploration of the issue will be necessary [47]. Additionally, throughout the paper, we have used a single waveform model, IMRPhenomHM [30] and checked for consistency with a more recently updated model IMRPhenomXHM [8]; but results are likely to vary somewhat with other models of the higher harmonics (for example, [35,38,39]). Finally, we have restricted attention throughout the paper to non-precessing systems. Recently, $[59,61]$, an analysis similar to the one presented here was performed on precessing systems, again with a focus on the observability of the two dominant harmonics. For systems where both higher harmonics and precession have an significant impact on the waveform, it will be necessary to combine these approaches to develop a straightforward categorization of precessing systems with observable higher harmonics. 


\section{ACKNOWLEDGMENTS}

The authors have benefitted from many valuable discussions with Edward Fauchon-Jones, Cecilio GarcíaQuirós, Rhys Green, Eleanor Hamilton, Mark Hannam, Charlie Hoy, Sebastian Khan, Lionel London, Frank Ohme, Vaibhav Tiwari and Vivien Raymond. S.F. and C.M. acknowledge support from the Science and Technology Facilities Council (STFC) Grant No. ST/L000962/1, European Research Council Consolidator Grant No. 647839ST/L000962/1. Finally, the authors are grateful for computational resources provided by the Cardiff University and LIGO Laboratory and supported by STFC Grant No. ST/I006285/1 and National Science Foundation Grants No. PHY-0757058 and No. PHY0823459 .

\section{APPENDIX A: SPIN-WEIGHTED SPHERICAL HARMONIC POLARIZATIONS}

The general form for the spin-weighted spherical harmonics is

$$
\begin{aligned}
{ }_{s} Y_{l m}\left(l, \phi_{o}\right)= & (-1)^{m} \sqrt{\frac{(l+m) !(l-m) !(2 l+1)}{4 \pi(l+s) !(l-s) !}} \sin ^{2 l}\left(\frac{l}{2}\right) \\
& \times \sum_{r=0}^{l-s}\left(\begin{array}{c}
l-s \\
r
\end{array}\right)\left(\begin{array}{c}
l+s \\
r+s-m
\end{array}\right) \\
& \times(-1)^{l-r-s} e^{i m \phi_{o}} \cot ^{2 r+s-m}\left(\frac{l}{2}\right),
\end{aligned}
$$

which can be written in terms of the Wigner d-functions $d_{-s}^{l m}(l)$ (implicitly defined here)

$$
{ }_{s} Y_{l m}\left(l, \phi_{o}\right)=\sqrt{\frac{(2 l+1)}{4 \pi}} d_{-s}^{l m}(l) e^{i m \phi_{o}} .
$$

They have the following symmetries

$$
\begin{aligned}
{ }_{s} \bar{Y}_{l m} & =(-1)^{s+m}{ }_{-s} Y_{l(-m)} \\
{ }_{s} Y_{l m}\left(\pi-\imath, \phi_{o}+\pi\right) & =(-1)_{-s}^{l} Y_{l m}\left(l, \phi_{o}\right) .
\end{aligned}
$$

The spin-weighted spherical harmonics for the harmonics we are interested in are

$$
\begin{aligned}
{ }_{2} Y_{22} & =\frac{1}{2} \sqrt{\frac{5}{\pi}} e^{i 2 \phi_{o}} \cos ^{4}\left(\frac{l}{2}\right) \\
{ }_{-2} Y_{2-2} & =\frac{1}{2} \sqrt{\frac{5}{\pi}} e^{-i 2 \phi_{o}} \sin ^{4}\left(\frac{l}{2}\right) \\
{ }_{2} Y_{21} & =\frac{1}{2} \sqrt{\frac{5}{\pi}} e^{i \phi_{o}} \cos ^{2}\left(\frac{l}{2}\right) \sin (l) \\
{ }_{2} Y_{2-1} & =\frac{1}{2} \sqrt{\frac{5}{\pi}} e^{-i \phi_{o}} \sin ^{2}\left(\frac{l}{2}\right) \sin (l) \\
{ }_{2} Y_{33} & =\frac{1}{2} \sqrt{\frac{21}{2 \pi}}\left(-e^{i 3 \phi_{o}}\right) \cos ^{4}\left(\frac{l}{2}\right) \sin (l) \\
{ }_{-2} Y_{3-3} & =\frac{1}{2} \sqrt{\frac{21}{2 \pi}} e^{-i 3 \phi_{o}} \sin ^{4}\left(\frac{l}{2}\right) \sin (l) \\
{ }_{2} Y_{32} & =\frac{1}{2} \sqrt{\frac{7}{\pi}} e^{i 2 \phi_{o}} \cos ^{4}\left(\frac{l}{2}\right)(3 \cos (l)-2) \\
{ }_{2} Y_{3-2} & =\frac{1}{2} \sqrt{\frac{7}{\pi}} e^{-i 2 \phi_{o}} \sin ^{4}\left(\frac{l}{2}\right)(3 \cos (l)+2) \\
{ }_{-2} Y_{44} & =\frac{3}{4} \sqrt{\frac{7}{\pi}} e^{i 4 \phi_{o}} \cos ^{4}\left(\frac{l}{2}\right) \sin ^{2}(l) \\
{ }_{2} Y_{4-4} & =\frac{3}{4} \sqrt{\frac{7}{\pi}} e^{-i 4 \phi_{o}} \sin ^{4}\left(\frac{l}{2}\right) \sin ^{2}(l) .
\end{aligned}
$$

We can write the gravitational wave polarizations as a sum of these spherical harmonics with coefficients $h_{l m}$

$$
h_{+}-i h_{\times}=\sum_{l \geq 2} \sum_{m=-l}^{l}{ }_{-2} Y_{l m}\left(l, \phi_{o}\right) h_{l m} .
$$

Three properties of $h_{l m}$ help to simplify Eq. (A3). First, specializing to planar (i.e., nonprecessing) binaries allows us to write $h_{l-m}=(-1)^{l} h_{l m}^{*}$ [1]. Second, in the frequency domain, $\tilde{h}_{l-m}^{*}(f)=\tilde{h}_{l m}(-f)^{*}$. Finally we make the further approximation [81] that if we only care about the waveform in direction $\hat{n}$ we can neglect one side of the frequency spectrum, depending on the sign of $m$. This approximation is valid in particular where the stationary phase approximation has been used. We therefore assume, with the sign convention on the Fourier transform as $\tilde{h}(f)=\int d t h(t) e^{+i 2 \pi f t}$, that

$$
\tilde{h}_{l m}(f) \simeq 0\left\{\begin{array}{l}
f>0, m<0 \\
f<0, m>0 .
\end{array}\right.
$$

With these three properties we can obtain explicit expressions for the orientation dependence of each of the harmonics for positive frequencies 


$$
\begin{aligned}
h_{+}= & \frac{1}{2} \sum_{l \geq 2} \sum_{m=-l}^{l}\left[_{-2} Y_{l m}\left(l, \phi_{o}\right) h_{l m}+{ }_{-2} Y_{l m}^{*}\left(l, \phi_{o}\right) h_{l m}^{*}\right] \\
\tilde{h}_{+}(f)= & \frac{1}{2} \sum_{l \geq 2} \sum_{m=-l}^{l}\left[{ }_{-2} Y_{l m}\left(l, \phi_{o}\right) \tilde{h}_{l m}(f)\right. \\
& \left.+{ }_{-2} Y_{l m}^{*}\left(l, \phi_{o}\right) \tilde{h}_{l m}(-f)^{*}\right] \\
= & \frac{1}{2} \sum_{l \geq 2} \sum_{m=1}^{l}\left[{ }_{-2} Y_{l m}\left(l, \phi_{o}\right)\right. \\
& \left.+(-1)_{-2}^{l} Y_{l-m}^{*}\left(l, \phi_{o}\right)\right] \tilde{h}_{l m}(f)
\end{aligned}
$$

and similarly we can show

$$
\begin{aligned}
h_{\times}= & \frac{i}{2} \sum_{l \geq 2} \sum_{m=-l}^{l}\left[_{-2} Y_{l m}\left(l, \phi_{o}\right) h_{l m}-{ }_{-2} Y_{l m}^{*}\left(l, \phi_{o}\right) h_{l m}^{*}\right] \\
\tilde{h}_{\times}(f)= & \frac{i}{2} \sum_{l \geq 2} \sum_{m=-l}^{l}\left[{ }_{-2} Y_{l m}\left(l, \phi_{o}\right) \tilde{h}_{l m}(f)\right. \\
& \left.-{ }_{-2} Y_{l m}^{*}\left(l, \phi_{o}\right) \tilde{h}_{l m}(-f)^{*}\right] \\
= & \frac{i}{2} \sum_{l \geq 2} \sum_{m=1}^{l}\left[{ }_{-2} Y_{l m}\left(l, \phi_{o}\right)\right. \\
& \left.-(-1)_{-2}^{l} Y_{l-m}^{*}\left(l, \phi_{o}\right)\right] \tilde{h}_{l m}(f) .
\end{aligned}
$$

where in both cases, we have neglected the $m=0$ terms in the sums as they are not considered in the models we have used. Finally, we note that we have used a different normalization convention in the main text, Eq. (3), than the one typically used in the spin-weighted spherical harmonic decomposition described in this Appendix. This has no impact on the results, but merely changes the values of $\alpha_{\ell m}$ and $R_{\ell m}$ while maintaining the same values of the SNR in the higher harmonics.

\section{APPENDIX B: DERIVATION OF $p\left(R_{l m}\right)$}

We now derive the probability distributions in Fig. 4. Assuming no preferred orientation for binaries in the universe, the probability density function for $\cos l$, $p(\cos l)$, is

$$
p_{\text {univ }}(\cos \imath)=\frac{1}{2}
$$

However, binaries which emit primarily in the 22 multipole radiate most powerfully in the direction perpendicular to the orbital plane, $|\cos \imath| \sim 1$. Consequently, the horizon for the subset of these binaries which are viewed edge-on is much closer and we preferentially observe face-on binaries. It can be shown [69] that the radiated power of the dominant multipole as a function of inclination is

$$
F(l)^{22}=\left(A_{+}^{22}\right)^{2}+\left(A_{\times}^{22}\right)^{2},
$$

where $A_{+, x}^{22}$ are defined in Eq. (4). For a detector sensitive to only one polarization of gravitational wave, the observed power will depend upon the polarization. This will also be the case for a network with different sensitivities to the two polarization, but not for one equally sensitive to both polarizations of the gravitational wave. It is possible to approximately marginalize over the polarization distribution and obtain a probability distribution for the inclinations of detected binaries, assuming sources are distributed uniformly in volume, as [69]

$p_{\text {det }}(\cos l) \propto F(l)^{3 / 2}=\left(1+6 \cos ^{2} \imath+\cos ^{4} l\right)^{3 / 2}$.

Using these results, it is straightforward to obtain expressions for the distributions for the expected power in the $(3,3)$ and $(4,4)$ multipoles, both for a uniform population of binaries and for those which are observable above a fixed threshold. The distribution for other multipoles can also be obtained but, since in general $R_{\ell m}$ will depend upon polarization angle, the results will be dependent upon the details of the network and its sensitivity to the two gravitational wave polarizations. For the $(3,3)$ and $(4,4)$ multipole moments, the relative amplitude depends only on the inclination angle $t$.

To obtain an expression for the probability distribution for $R_{\ell m}$, we change variables

$$
p\left(R_{\ell m}\right)=\left(\frac{d \cos \iota}{d R_{\ell m}}\right) p(\cos \iota)
$$

so that, recalling the functional form of $R_{33}$ and $R_{44}$ from Eq. (15), we obtain

$$
\begin{aligned}
& p_{\text {univ }}\left(R_{33}\right)=\frac{R_{33}}{4 \sqrt{1-\left(\frac{R_{33}}{2}\right)^{2}}} \\
& p_{\text {univ }}\left(R_{44}\right)=\frac{1}{4 \sqrt{1-\left(\frac{R_{44}}{2}\right)}} .
\end{aligned}
$$

Assuming binaries are detected with $(2,2)$ harmonic-only waveforms, we can apply the same weighting factor as above in obtaining the distributions for the observed binaries, to obtain

$$
p_{\text {det }}\left(R_{\ell m}\right)=\left(\frac{d \cos \imath}{d R_{\ell m}}\right) p_{\text {det }}(\cos \imath)
$$

which gives

$$
\begin{aligned}
& p_{\text {det }}\left(R_{33}\right) \propto\left(8-2 R_{33}^{2}+\frac{R_{33}^{4}}{16}\right) p_{\text {univ }}\left(R_{33}\right) \\
& p_{\text {det }}\left(R_{44}\right) \propto\left(8-4 R_{44}+\frac{R_{44}^{2}}{4}\right) p_{\text {univ }}\left(R_{44}\right)
\end{aligned}
$$

These distributions are plotted in Fig. 4, and discussed in the surrounding text. 
[1] L. Blanchet, Gravitational radiation from post-Newtonian sources and inspiralling compact binaries, Living Rev. Relativity 17, 2 (2014).

[2] E. T. Newman and R. Penrose, Note on the Bondi-MetznerSachs group, J. Math. Phys. (N.Y.) 7, 863 (1966).

[3] K. Thorne, Multipole expansions of gravitational radiation, Rev. Mod. Phys. 52, 299 (1980).

[4] S. A. Teukolsky, Perturbations of a rotating black hole. 1. Fundamental equations for gravitational electromagnetic and neutrino field perturbations, Astrophys. J. 185, 635 (1973).

[5] E. Leaver, An analytic representation for the quasi normal modes of Kerr black holes, Proc. R. Soc. A 402, 285 (1985).

[6] S. A. Hughes, The evolution of circular, nonequatorial orbits of Kerr black holes due to gravitational wave emission, Phys. Rev. D 61, 084004 (2000); Erratum, Phys. Rev. D 63, 049902 (2001); Erratum, Phys. Rev. D 65, 069902(E) (2002); Erratum, Phys. Rev. D 67, 089901(E) (2003); Erratum, Phys. Rev. D 78, 109902(E) (2008); Erratum, Phys. Rev. D 90, 109904(E) (2014).

[7] L. London, Notes on the spheroidal harmonic multipole moments of gravitational radiation, arXiv:2006.11449.

[8] C. García-Quirós, M. Colleoni, S. Husa, H. Estellés, G. Pratten, A. Ramos-Buades, M. Mateu-Lucena, and R. Jaume, Multimode frequency-domain model for the gravitational wave signal from nonprecessing black-hole binaries, Phys. Rev. D 102, 064002 (2020).

[9] L. E. Kidder, Using full information when computing modes of post-Newtonian waveforms from inspiralling compact binaries in circular orbit, Phys. Rev. D 77, 044016 (2008).

[10] C. K. Mishra, A. Kela, K. G. Arun, and G. Faye, Ready-touse post-Newtonian gravitational waveforms for binary black holes with nonprecessing spins: An update, Phys. Rev. D 93, 084054 (2016).

[11] E. Berti, V. Cardoso, J. A. Gonzalez, U. Sperhake, M. Hannam, S. Husa, and B. Bruegmann, Inspiral, merger and ringdown of unequal mass black hole binaries: A multipolar analysis, Phys. Rev. D 76, 064034 (2007).

[12] L. E. Kidder, Using full information when computing modes of post-Newtonian waveforms from inspiralling compact binaries in circular orbit, Phys. Rev. D 77, 044016 (2008).

[13] L. Blanchet, G. Faye, B. R. Iyer, and S. Sinha, The third post-Newtonian gravitational wave polarizations and associated spherical harmonic modes for inspiralling compact binaries in quasi-circular orbits, Classical Quantum Gravity 25, 165003 (2008).

[14] J. Healy, P. Laguna, L. Pekowsky, and D. Shoemaker, Template mode hierarchies for binary black hole mergers, Phys. Rev. D 88, 024034 (2013).

[15] L. Blanchet, Gravitational radiation from post-Newtonian sources and inspiralling compact binaries, Living Rev. Relativity 9, 4 (2006).

[16] L. London, D. Shoemaker, and J. Healy, Modeling ringdown: Beyond the fundamental quasinormal modes, Phys. Rev. D 90, 124032 (2014).

[17] L. Pekowsky, J. Healy, D. Shoemaker, and P. Laguna, Impact of higher-order modes on the detection of binary black hole coalescences, Phys. Rev. D 87, 084008 (2013).

[18] J. C. Bustillo, S. Husa, A. M. Sintes, and M. Pürrer, Impact of gravitational radiation higher order modes on single aligned-spin gravitational wave searches for binary black holes, Phys. Rev. D 93, 084019 (2016).

[19] J. C. Bustillo, P. Laguna, and D. Shoemaker, Detectability of gravitational waves from binary black holes: Impact of precession and higher modes, Phys. Rev. D 95, 104038 (2017).

[20] P. B. Graff, A. Buonanno, and B. S. Sathyaprakash, Missing link: Bayesian detection and measurement of intermediatemass black-hole binaries, Phys. Rev. D 92, 022002 (2015).

[21] Y. Pan, A. Buonanno, M. Boyle, L. T. Buchman, L. E. Kidder, H. P. Pfeiffer, and M. A. Scheel, Inspiral-mergerringdown multipolar waveforms of nonspinning black-hole binaries using the effective-one-body formalism, Phys. Rev. D 84, 124052 (2011).

[22] E. Berti, J. Cardoso, V. Cardoso, and M. Cavaglia, Matchedfiltering and parameter estimation of ringdown waveforms, Phys. Rev. D 76, 104044 (2007).

[23] I. Harry, J. C. Bustillo, and A. Nitz, Searching for the full symphony of black hole binary mergers, Phys. Rev. D 97, 023004 (2018).

[24] C. Capano, Y. Pan, and A. Buonanno, Impact of higher harmonics in searching for gravitational waves from nonspinning binary black holes, Phys. Rev. D 89, 102003 (2014).

[25] V. Varma, P. Ajith, S. Husa, J. C. Bustillo, M. Hannam, and M. Prrer, Gravitational-wave observations of binary black holes: Effect of nonquadrupole modes, Phys. Rev. D 90, 124004 (2014).

[26] C. Van Den Broeck and A. S. Sengupta, Binary black hole spectroscopy, Classical Quantum Gravity 24, 1089 (2007).

[27] K. G. Arun, A. Buonanno, G. Faye, and E. Ochsner, Higherorder spin effects in the amplitude and phase of gravitational waveforms emitted by inspiraling compact binaries: Readyto-use gravitational waveforms, Phys. Rev. D 79, 104023 (2009).

[28] I. Kamaretsos, M. Hannam, S. Husa, and B. S. Sathyaprakash, Black-hole hair loss: Learning about binary progenitors from ringdown signals, Phys. Rev. D 85, 024018 (2012).

[29] A. K. Mehta, C. K. Mishra, V. Varma, and P. Ajith, Accurate inspiral-merger-ringdown gravitational waveforms for nonspinning black-hole binaries including the effect of subdominant modes, Phys. Rev. D 96, 124010 (2017).

[30] L. London, S. Khan, E. Fauchon-Jones, C. Garca, M. Hannam, S. Husa, X. Jimnez-Forteza, C. Kalaghatgi, F. Ohme, and F. Pannarale, First Higher-Multipole Model of Gravitational Waves from Spinning and Coalescing BlackHole Binaries, Phys. Rev. Lett. 120, 161102 (2018).

[31] S. Khan, K. Chatziioannou, M. Hannam, and F. Ohme, Phenomenological model for the gravitational-wave signal from precessing binary black holes with two-spin effects, Phys. Rev. D 100, 024059 (2019).

[32] S. Khan, F. Ohme, K. Chatziioannou, and M. Hannam, Including higher order multipoles in gravitational-wave models for precessing binary black holes, Phys. Rev. D 101, 024056 (2020).

[33] A. K. Mehta, P. Tiwari, N. K. Johnson-McDaniel, C. K. Mishra, V. Varma, and P. Ajith, Including mode mixing in a higher-multipole model for gravitational waveforms from 
nonspinning black-hole binaries, Phys. Rev. D 100, 024032 (2019).

[34] R. Cotesta, A. Buonanno, A. Boh, A. Taracchini, I. Hinder, and S. Ossokine, Enriching the symphony of gravitational waves from binary black holes by tuning higher harmonics, Phys. Rev. D 98, 084028 (2018).

[35] V. Varma, S. E. Field, M. A. Scheel, J. Blackman, L. E. Kidder, and H. P. Pfeiffer, Surrogate model of hybridized numerical relativity binary black hole waveforms, Phys. Rev. D 99, 064045 (2019).

[36] V. Varma, S. E. Field, M. A. Scheel, J. Blackman, D. Gerosa, L. C. Stein, L. E. Kidder, and H. P. Pfeiffer, Surrogate models for precessing binary black hole simulations with unequal masses, Phys. Rev. Research 1, 033015 (2019).

[37] N. E. M. Rifat, S. E. Field, G. Khanna, and V. Varma, A surrogate model for gravitational wave signals from comparable- to large- mass-ratio black hole binaries, Phys. Rev. D 101, 081502 (2020).

[38] A. Nagar, G. Riemenschneider, G. Pratten, P. Rettegno, and F. Messina, A multipolar effective one body waveform model for spin-aligned black hole binaries, Phys. Rev. D 102, 024077 (2020).

[39] R. Cotesta, S. Marsat, and M. Pürrer, Frequency domain reduced order model of aligned-spin effective-one-body waveforms with higher-order modes, Phys. Rev. D 101, 124040 (2020).

[40] S. Ossokine, A. Buonanno, S. Marsat, R. Cotesta, S. Babak, T. Dietrich, R. Haas, I. Hinder, H. P. Pfeiffe, M. Pürrer et al., Technical Report No. LIGO-P2000140, 2020.

[41] G. Pratten et al., Let's twist again: Computationally efficient models for the dominant and sub-dominant harmonic modes of precessing binary black holes, arXiv:2004.06503.

[42] R. Abbott et al. (LIGO Scientific and Virgo Collaborations), GW190412: Observation of a binary-black-hole coalescence with asymmetric masses, Phys. Rev. D 102, 043015 (2020).

[43] R. Abbott et al. (LIGO Scientific and Virgo Collaborations), GW190814: Gravitational waves from the coalescence of a 23 solar mass black hole with a 2.6 solar mass compact object, Astrophys. J. 896, L44 (2020).

[44] K. Chatziioannou, R. Cotesta, S. Ghonge, J. Lange, K. K. Y. Ng, J. C. Bustillo, J. Clark, C.-J. Haster, S. Khan, M. Pürrer et al., On the properties of the massive binary black hole merger GW170729, Phys. Rev. D 100, 104015 (2019).

[45] C. Van Den Broeck and A. S. Sengupta, Phenomenology of amplitude-corrected post-Newtonian gravitational waveforms for compact binary inspiral. I. Signal-to-noise ratios, Classical Quantum Gravity 24, 155 (2007).

[46] B. P. Abbott, R. Abbott, T. D. Abbott, S. Abraham, F. Acernese, K. Ackley, C. Adams, R. X. Adhikari, V. B. Adya, C. Affeldt et al., Binary black hole population properties inferred from the first and second observing runs of Advanced LIGO and Advanced Virgo, Astrophys. J. 882, L24 (2019).

[47] C. Kalaghatgi, M. Hannam, and V. Raymond, Parameter Estimation with a spinning multi-mode waveform model: IMRPhenomHM, Phys. Rev. D 101, 103004 (2020).
[48] S. A. Usman, J. C. Mills, and S. Fairhurst, Constraining the inclinations of binary mergers from gravitational-wave observations, Astrophys. J. 877, 82 (2019).

[49] F. Ohme, A. B. Nielsen, D. Keppel, and A. Lundgren, Statistical and systematic errors for gravitational-wave inspiral signals: A principal component analysis, Phys. Rev. D 88, 042002 (2013).

[50] M. Hannam, D. A. Brown, S. Fairhurst, C. L. Fryer, and I. W. Harry, When can gravitational-wave observations distinguish between black holes and neutron stars?, Astrophys. J. 766, L14 (2013).

[51] T. A. Apostolatos, C. Cutler, G. J. Sussman, and K. S. Thorne, Spin induced orbital precession and its modulation of the gravitational wave forms from merging binaries, Phys. Rev. D 49, 6274 (1994).

[52] R. Green, C. Hoy, S. Fairhurst, M. Hannam, F. Pannarale, and C. Thomas, Identifying when precession can be measured in gravitational waveforms, arXiv:2010.04131.

[53] A. Bauswein and H. T. Janka, Measuring neutron-star properties via gravitational waves from binary mergers, Phys. Rev. Lett. 108, 011101 (2012).

[54] K. Chatziioannou, C.-J. Haster, and A. Zimmerman, Measuring the neutron star tidal deformability with equation-ofstate-independent relations and gravitational waves, Phys. Rev. D 97, 104036 (2018).

[55] S. De, D. Finstad, J. M. Lattimer, D. A. Brown, E. Berger, and C.M. Biwer, Tidal Deformabilities and Radii of Neutron Stars from the Observation of GW170817, Phys. Rev. Lett. 121, 091102 (2018); , Erratum, Phys. Rev. Lett. 121, 259902 (2018).

[56] B. P. Abbott, R. Abbott, T. D. Abbott, F. Acernese, K. Ackley, C. Adams, T. Adams, P. Addesso, R. X. Adhikari, V. B. Adya et al., GW170817: Measurements of Neutron Star Radii and Equation of State, Phys. Rev. Lett. 121, 161101 (2018).

[57] R. Abbott et al. (LIGO Scientific and Virgo Collaborations), GW190521: A Binary Black Hole Merger with a Total Mass of $150 M_{\odot}$, Phys. Rev. Lett. 125, 101102 (2020).

[58] M. Hannam, P. Schmidt, A. Bohé, L. Haegel, S. Husa, F. Ohme, G. Pratten, and M. Pürrer, Simple Model of Complete Precessing Black-Hole-Binary Gravitational Waveforms, Phys. Rev. Lett. 113, 151101 (2014).

[59] S. Fairhurst, R. Green, C. Hoy, M. Hannam, and A. Muir, The two-harmonic approximation for gravitational waveforms from precessing binaries, Phys. Rev. D 102, 024055 (2020).

[60] R. Abbott et al. (LIGO Scientific and Virgo Collaborations), GWTC-2: Compact Binary Coalescences Observed by LIGO and Virgo During the First Half of the Third Observing Run, arXiv:2010.14527.

[61] S. Fairhurst, R. Green, M. Hannam, and C. Hoy, When will we observe binary black holes precessing?, Phys. Rev. D 102, 041302 (2020).

[62] S. Klimenko, S. Mohanty, M. Rakhmanov, and G. Mitselmakher, Constraint likelihood analysis for a network of gravitational wave detectors, Phys. Rev. D 72, 122002 (2005).

[63] I. W. Harry and S. Fairhurst, A targeted coherent search for gravitational waves from compact binary coalescences, Phys. Rev. D 83, 084002 (2011). 
[64] M. Boyle, R. Owen, and H. P. Pfeiffer, A geometric approach to the precession of compact binaries, Phys. Rev. D 84, 124011 (2011).

[65] E. Berti, V. Cardoso, and C. M. Will, On gravitational-wave spectroscopy of massive black holes with the space interferometer LISA, Phys. Rev. D 73, 064030 (2006).

[66] S. Roy, A.S. Sengupta, and K. Arun, Unveiling the spectrum of inspiralling binary black holes, arXiv:1910 .04565 .

[67] M. Evans et al., Technical Report No. LIGO-T1500293, 2020, https://dcc.ligo.org/LIGO-T1500293.

[68] P. Madau and M. Dickinson, Cosmic star-formation history, Annu. Rev. Astron. Astrophys. 52, 415 (2014).

[69] B. F. Schutz, Networks of gravitational wave detectors and three figures of merit, Classical Quantum Gravity 28, 125023 (2011).

[70] S. A. Usman, A. H. Nitz, I. W. Harry, C. M. Biwer, D. A. Brown, M. Cabero, C. D. Capano, T. Dal Canton, T. Dent, S. Fairhurst et al., The PyCBC search for gravitational waves from compact binary coalescence, Classical Quantum Gravity 33, 215004 (2016).

[71] A. H. Nitz, T. Dent, T. Dal Canton, S. Fairhurst, and D. A. Brown, Detecting binary compact-object mergers with gravitational waves: Understanding and improving the sensitivity of the PyCBC search, Astrophys. J. 849, 118 (2017).

[72] C. Hanna, S. Caudill, C. Messick, A. Reza, S. Sachdev, L. Tsukada, K. Cannon, K. Blackburn, J. D. E. Creighton, H. Fong et al., Fast evaluation of multidetector consistency for real-time gravitational wave searches, Phys. Rev. D 101, 022003 (2020).
[73] J. Veitch et al., Parameter estimation for compact binaries with ground-based gravitational-wave observations using the LALInference software library, Phys. Rev. D 91, 042003 (2015).

[74] C. Cutler and E. E. Flanagan, Gravitational waves from merging compact binaries: How accurately can one extract the binary's parameters from the inspiral wave form?, Phys. Rev. D 49, 2658 (1994).

[75] E. E. Flanagan and S. A. Hughes, Measuring gravitational waves from binary black hole coalescences: 2 . The waves' information and its extraction, with and without templates, Phys. Rev. D 57, 4566 (1998).

[76] M. A. Miller, Accuracy requirements for the calculation of gravitational waveforms from coalescing compact binaries in numerical relativity, Phys. Rev. D 71, 104016 (2005).

[77] L. Lindblom, B. J. Owen, and D. A. Brown, Model waveform accuracy standards for gravitational wave data analysis, Phys. Rev. D 78, 124020 (2008).

[78] E. Baird, S. Fairhurst, M. Hannam, and P. Murphy, Degeneracy between mass and spin in black-hole-binary waveforms, Phys. Rev. D 87, 024035 (2013).

[79] S. Bhagwat, D. A. Brown, and S. W. Ballmer, Spectroscopic analysis of stellar mass black-hole mergers in our local universe with ground-based gravitational wave detectors, Phys. Rev. D 94, 084024 (2016); , Erratum, Phys. Rev. D 95, 069906 (2017).

[80] V. Tiwari, S. Fairhurst, and M. Hannam, Constraining blackhole spins with gravitational wave observations, Astrophys. J. 868, 140 (2018).

[81] S. Marsat and J. G. Baker, Fourier-domain modulations and delays of gravitational-wave signals, arXiv:1806.10734. 